\title{
Polarly localized receptor-like kinases PXC2 and IRK act redundantly during Arabidopsis root development in the radial axis
}

Jason Goff and Jaimie M. Van Norman*

Department of Botany and Plant Sciences, Center for Plant Cell Biology, Institute of Integrative Genome Biology, University of California, Riverside, USA

*Corresponding author: Jaimie M. Van Norman, jaimie.vannorman@ucr.edu

\section{RUNNING HEAD}

Polar localized PXC2 functions in root development

\section{KEYWORDS}

Root development, cell division, receptor kinase, polar localization, endodermis, Arabidopsis root

\section{SUMMARY}

In plants, coordination of cell division and differentiation is critical for tissue patterning and organ development. Directional cell signaling and cell polarity have been proposed to participate in coordination of these developmental processes. For instance, a leucine-rich repeat receptor-like kinase (LRR-RLK) named INFLORESCENCE AND ROOT APICES KINASE (IRK) functions to restrict stele area and inhibit longitudinal anticlinal divisions (LADs) in the endodermis where it is polarly localized. The LRR-RLK most closely related to IRK is PXY/TDR CORRELATED 2 (PXC2) and we find that PXC2 shows similar polarized accumulation as IRK in root cell types. To further understand how these proteins operate in directional cell-cell signaling and root development we explored PXC2 function. pxc2 roots have an increase in stele area, indicating that PXC2 also functions to restrict stele size. Additionally, compared to either single mutant, irk pxc2 roots have an enhanced phenotype with further increases in endodermal LADs and stele area indicating redundant activities of these receptors. The double mutant also exhibits abnormal root growth, suggesting broader functions of PXC2 and IRK in the root. However, PXC2 is not functionally equivalent to IRK, as endodermal misexpression of $P X C 2$ did not fully rescue irk. We propose that PXC2 is at least partially redundant to IRK with a more predominant role for IRK in repression of endodermal LADs. Our results are consistent with the hypothesis that repression of specific endodermal cell divisions and stele area through a PXC2/IRKmediated directional signaling pathway is required for coordinated root growth and development. 


\section{SIGNIFICANCE STATEMENT}

In the root, coordination of growth and developmental processes is critical for organ function and directional cell-to-cell signaling and cell polarity are implicated in these processes. Our studies indicate that laterally polar transmembrane receptor kinases, PXC2 and IRK, have redundant functions in restriction of specific endodermal cell divisions and stele size, and are important for gravitropic root growth.

\section{INTRODUCTION}

In plant development, proper orientation of cell divisions relative to the body axis is required for tissue and organ patterning and overall organ shape (Shao and Dong, 2016; Martinez et al., 2017; Facette et al., 2018; Rasmussen and Bellinger, 2018). Defects in tissue patterning and organ shape can negatively impact organ function (Meyerowitz, 1997; Camilleri et al., 2002). The organization of root tissues and cell types is critical to its function in anchorage and uptake and can be partly attributed to strict regulation of the orientation of root cell divisions. In the root, cell divisions are typically oriented periclinally (parallel to the root's surface) or anticlinally (perpendicular to the root's surface) which generates more cells in the radial and/or longitudinal axes. The highly stereotypical organization of root cell types and tissues makes them an ideal model in which to investigate the mechanisms underlying coordination of oriented cell division and tissue patterning during organ development.

In the Arabidopsis root, the central stele, which contains the pericycle and the vascular tissues, is surrounded by the endodermis, cortex, and epidermis in the transverse axis (Figure 1A) (Dolan et al., 1993). In the longitudinal axis, cell types are maintained in linear files that extend from the stem cell niche shootward. For example, in the root ground tissue, the cortex and endodermal cell types are derived from a single stem (initial) cell, the cortex/endodermal initial (CEI). The CEI divides to produce a daughter cell which then undergoes a periclinal cell division to produce a pair of cell types: the endodermis towards the inside and the cortex towards the outside (Figure 1A) (Dolan et al., 1993; Scheres and Benfey, 1999). Within the root meristem, cells proliferate in longitudinal files extending shootward through transverse anticlinal divisions. In the root ground tissue, longitudinal anticlinal cell divisions rarely occur in the meristem with these divisions producing more ground tissue cells around the stele (Campos et al., 2020). Following proliferation in the meristem, cells undergo dramatic elongation which accounts for the majority of root lengthening (Hodge et al., 2009). In order for the root to maintain its ability to effectively navigate a dynamic and heterogeneous soil environment, precise cellular organization together with coordinated cell elongation must be maintained. Differential cell elongation within the root allows for directional root growth due to positive and negative trophic responses to various aspects of their environment (Correll and Kiss, 2005; Dyson et al., 2014; Dietrich et al., 2017; Su et al., 2017). Thus, disrupting the root's ability to coordinate cell division and/or cell elongation can lead to defects in root form and function. 
Coordination of cellular processes, including division and elongation, in any plant organ requires cell-cell communication (Van Norman et al., 2011; Chaiwanon et al., 2016; Wu et al., 2016). Furthermore, orientation of cell divisions and cell fate specification cues are proposed to be based largely on extrinsic factors. This model is supported by cell ablation studies in the root meristem that show altered cell division patterns and cell fate specification when neighboring cells are lost and replaced through division of internal neighbors (van den Burg et al., 1995; van den Berg et al., 1997; Marhava et al., 2019). In plants, a large family of receptor-like kinases (RLKs) is proposed to serve as major participants in intercellular communication across the plant body. This protein family has three main subgroups with the transmembrane group of RLKs containing over 200 members, each with a series of extracellular leucine-rich repeats (LRRs), a single transmembrane domain, and a cytoplasmic kinase domain (Shiu and Bleecker, 2001, 2003). These LRR-RLKs are generally predicted to perceive extracellular ligands and activate downstream signaling pathways. However, many LRR-RLKs remain uncharacterized partially due to high levels of functional redundancy and/or very specific or mild mutant phenotypes (Diévart and Clark, 2003).

In the Arabidopsis root the LRR-RLK, IRK, is polarly localized to the outer (lateral) polar domain in endodermal cells; yet, in other root cell types, it is localized to distinct domains or is nonpolar (Campos et al., 2020). irk mutants have excess longitudinal anticlinal and periclinal endodermal cell divisions and show increased stele area in the radial axis. These defects are rescued by expression of IRK from its endogenous promoter or by endodermal-specific expression of IRK, suggesting that abnormal LAD of endodermal cells is the primary defect in irk mutants (Campos et al., 2020). Despite the cellular level abnormalities in irk, root growth and overall morphology appear largely normal. This led us to investigate whether a related LRR-RLK was obscuring a more detrimental dysregulation of endodermal LADs and an enhanced impact on root growth and development.

IRK is most closely related to PXY/TDR-CORRELATED 2 (PXC2, encoded by At5g01890) (Shiu and Bleecker, 2001, 2003; Wang et al., 2013). PXC2 was recently also given the name CANALIZATION-RELATED RECEPTOR-LIKE KINASE (CANAR) and shown to coordinate PIN-FORMED 1 (PIN1) localization during auxin canalization with canar mutants having defects in cotyledon and leaf venation and vascular regeneration after wounding (Hajný et al., 2020). As PXC2 and IRK are both expressed in the root tip (Wang et al., 2013; Campos et al., 2020), we hypothesized that these LRR-RLKs have similar and/or overlapping functions in root development.

Here we show that PXC2 and IRK are present in the same root cell types and, like IRK, PXC2 is localized to the outer (lateral) polar domain in the endodermis and to distinct polar domains or is nonpolar in other cell types. Unlike irk mutants, which show increases in both stele area and endodermal LADs, we find that pxc2 single mutant roots only show increased stele area. However, irk pxc2 double mutant roots exhibit a further increase in both endodermal LADs and stele area, beyond either single mutant, indicating that polarly localized IRK and PXC2 function redundantly to repress endodermal LADs and stele area during root development. The double mutant also exhibits an abnormal root growth phenotype not present 
in either single mutant, suggesting enhanced dysregulation of endodermal LADs and stele size negatively impact overall root growth or that IRK and PXC2 are redundantly required for root gravitropism. Finally, we show that PXC2 can only partially rescue the irk root phenotypes, indicating PXC2 is not functionally equivalent to IRK. We propose these polarized receptors function redundantly in a directional signaling pathway in the root required for coordination of oriented cell divisions, tissue patterning, and growth.

\section{RESULTS}

\section{PXC2-GFP is polarly localized root tissues}

To examine the expression and localization of PXC2, we first generated a transcriptional fusion using the entire intergenic region (4.7 kilobases (kb)) upstream of the PXC2 start codon $(p P X C 2)$ to drive expression of endoplasmic reticulum-localized green fluorescent protein (erGFP). In wild type roots, we observed that $p P X C 2$ activity is restricted to the lateral root cap and xylem cell types extending shootward from cells adjacent to the quiescence center (QC) (Figure S1A, B). This is largely consistent with previous examination of $p P X C 2$ activity via a promoter-driven GUS ( $\beta$-glucuronidase) reporter (Wang et al., 2013), however, this pPXC2:GUS reporter contains just $1.9 \mathrm{~kb}$ of the intergenic sequence upstream of PXC2 ((Wang et al., 2013); Dr. Bo Zhang, personal communication). Thus, any differences between these reporters may be due to examination of $p P X C 2$ :erGFP at higher cellular resolution or to differences in promoter length. These results indicate that $p P X C 2$ is most active in the LRC and xylem cell types.

To examine PXC2 accumulation, we expressed PXC2 fused to GFP under $p P X C 2$ ( $p P X C 2: P X C 2: G F P$ ) and observed low levels of the fusion protein in many root cell types with stronger accumulation in the plasma membrane of the LRC and xylem cell types (Figure 1B and S1C-E). Additionally, PXC2-GFP is weakly detectable in other root cell types beyond where pPXC2 activity is detected (compare Figures $1 \mathrm{~B}$ and S1A, D). Like IRK-GFP, the accumulation of PXC2-GFP appeared polarly distributed in the plasma membrane, particularly at the inner polar domain of the outermost layer of the LRC (Figure 1B). However, in the xylem cell types, PXC2-GFP appears to have non-polar accumulation (Figures 1B, G and S1C). Due to very low GFP signal in most root cell types, it was particularly difficult to determine whether accumulation of PXC2-GFP was polarly localized in multiple root cell types.

Despite being driven by the same promoter, our PXC2 transcriptional and translational reporters show only partially overlapping patterns of expression. Accumulation of the PXC2GFP fusion protein in cell types beyond those where $p P X C 2$ :erGFP is detected may be due to very low levels of $p P X C 2$ activity, which fails to produce enough erGFP for detection. However, when $p P X C 2$ drives PXC2-GFP expression, this fusion protein is detectable due to its accumulation at the plasma membrane. Additionally, expression of $p P X C 2: P X C 2: G F P$ rescues the enhanced irk pxc2 double mutant back to the irk single mutant phenotype (see below, Figure S4C, D), suggesting the accumulation and function of the $p P X C 2: P X C 2: G F P$ transgene replicates the endogenous situation. 
To determine whether PXC2 has similar polarized accumulation as IRK, we investigated PXC2-GFP localization using root cell- or tissue-specific promoters to drive its expression. Because $p P X C 2$ is highly active in the LRC, we expressed PXC2-GFP from the SOMBRERO promoter ( $p S M B)$, which is expressed specifically in these cells (Willemsen et al., 2008). Expression with $p S M B$ confirmed localization of PXC2-GFP to the inner polar domain in the LRC (Figure 1C). Additionally, PXC2-GFP expressed with $p W E R E W O L F(p W E R)$ in the LRC and epidermis (Lee and Schiefelbein, 1999) and pCORTEX2 (pCO2) in the cortex (Sabatini et al., 2003; Paquette and Benfey, 2005) is also localized to the inner polar domain (Figure 1D, E). Furthermore, similar to IRK, when expressed from pSCARECROW ( $p S C R$ ) (Wysocka-Diller et al., 2000), PXC2-GFP localizes to the outer polar domain in the endodermis and the root- and shootward polar domains in the CEI (Figure 1F). Cell type/layer-specific expression allowed PXC2-GFP accumulation to be examined with less interference from signal in surrounding cells (Alassimone et al., 2010; Campos et al., 2020). Together, these results indicate that, like IRK, PXC2 is a polarly localized LRR-RLK that accumulates to different polar domains in different cell types (Figure 1G). The overlapping expression and polar accumulation of IRK and PXC2 are consistent with our hypothesis that they have overlapping or redundant functions in the root.

\section{Mutant alleles of PXC2 have an enlarged stele area}

To study PXC2 function during root development, two insertion alleles were examined, SM_3_31635 (pxc2-3) and Salk_055351 (pxc2-1/canar-1). We determined that the insertion site in pxc2-3 resides in the second exon upstream of the sequence encoding the transmembrane domain and generates a premature stop codon 10 codons after the insertion site (Figure S2A). $P X C 2$ transcript accumulation is reduced in pxc2-3 (Figure S2B) and based on the position of the insertion, $p x c 2-3$ is unlikely to produce functional protein making it a likely null allele. The insertion in pxc2-1/canar-1 is also located in the second exon but is downstream of the sequence encoding the transmembrane domain (Figure S2A), suggesting a truncated protein could be formed. Additionally, we did not find a significant reduction in PXC2 transcript in pxc21/canar-1 when compared to wild type (Figure S2B). The pxc2-2/canar-2 (Salk_018730) allele was not examined here due to the availability of other alleles that, based on the T-DNA position, we predicted were more likely to be loss of function alleles.

No abnormal root phenotype was detected when pxc2-3 seedlings were grown on our standard media (not shown). In an attempt to identify an abnormal root phenotype, we grew these seedlings on media containing $0.2 x$ Murashige and Skoog (0.2x MS) basal salts, which increases root growth due to lower levels of nitrogen and potassium but does not alter patterning. Under these conditions, we observed an increase in stele area, but no excess endodermal LADs in pxc2-3 compared to Col-0 (Figure 2A-D). A similar phenotype was also observed in pxc2-1/canar-1 roots (Figure S3A-F), indicating that both alleles disrupt $P X C 2$ function. These results indicate PXC2 functions to restrict stele area. However, because an abnormal phenotype was detected only on media containing $0.2 x \mathrm{MS}, \mathrm{PXC} 2$ may serve a relatively minor role in this process or its role may be dependent on growth conditions. Thus, pxc2 mutants exhibit a subset of the phenotypes observed in irk-4 roots, which show increased 
stele area and excess endodermal LADs (Campos et al., 2020). Additionally, IRK expression is upregulated in the pxc2-1/canar-1 mutant background, but no significant change in $P X C 2$ transcript in the irk-4 mutant was observed (Figure S2C, D). These results are consistent with IRK and PXC2 having overlapping or redundant functions in the root.

\section{PXC2 and IRK have redundant functions in restricting stele area and repressing endodermal longitudinal anticlinal cell divisions}

To test for functional redundancy, irk-4 pxc2-3 double mutants were generated by standard genetic crosses. When compared to the single mutants, irk-4 pxc2-3 roots at 4 dps display an enhanced abnormal phenotype with a highly disorganized endodermis, including many additional LADs and an increased enlargement of stele area (Figure 2E-H). Similar to irk4 , the excess LADs often occur in endodermal cells adjacent to the xylem axis (Figures $2 \mathrm{~F}$ and S4B). At 6 dps, the cell division phenotypes in irk-4 pxc2-3 roots are substantially more severe (Figure S4A, B). This made it difficult to confidently identify whether a cell was the direct product of an endodermal LAD or periclinal division, so quantification was deemed unreliable in older seedlings. To ensure that the phenotypic enhancement observed in irk-4 pxc2-3 double mutants was due to simultaneous loss-of-function of both genes and not to disruption of any other locus, we expressed the $p P X C 2: P X C 2: G F P$ in the irk-4 pxc2-3 background and observed rescue of the double mutant phenotype to the level of the irk-4 single mutant (Figure S4C, D). These results indicate that the enhanced irk pxc2 double mutant phenotype is specific to the loss of IRK and PXC2 function.

Enlarged stele area in irk and pxc2 single mutants, suggests both proteins are required to restrict its size. It is possible that IRK and PXC2 have independent functions in this process, however, these proteins may also function redundantly with a lower dosage of the gene products leading to weaker single mutant phenotypes. Although $p x c 2$ single mutants do not have excess endodermal LADs, the irk pxc2 double mutant has a greater number of these divisions than irk alone; thus, we propose that IRK is fully redundant with PXC2 to prevent these divisions from occurring. Given the enhanced phenotypic severity in the double mutant, in terms of both endodermal LADs and stele area, we conclude that IRK and PXC2 have redundant functions to negatively regulate these processes.

\section{irk pxc2 double mutants exhibit a root growth defect}

While investigating the cellular phenotypes in irk-4 pxc2-3 roots, we observed a root growth phenotype not present in either single mutant. irk-4 pxc2-3 roots are somewhat shorter and do not grow as consistently and uniformly downward as wild type, appearing to have a somewhat agravitropic growth phenotype (Figure 3B-D). When compared to wild type, irk pxc2 mutants show reduced root straightness (Figure $3 \mathrm{~A}, \mathrm{E}$ ), as assessed by the ratio of the distance from hypocotyl to root tip (Dy) divided by total root length. In some replicates irk-4 root length was slightly increased, but these results were not consistently replicated; thus, root length and straightness were not significantly altered in pxc2-3 and irk-4 single mutants (Figure S4E-H). Furthermore, upon reorientation of the seedlings with respect to the gravity vector, irk-4 pxc2-3 mutant roots show an attenuated gravitropic response compared to wild type, while the single 
mutants showed normal responses (Figures 3F and S4I, J). Additionally, in contrast to WT roots, which exhibit a weak right-slanting (Dx) growth habit (Grabov et al., 2005; ArribasHernández et al., 2020), all mutant genotypes show a left-slanting growth habit (Figures 3A, GI). Because increased stele area and left-slanted growth are observed in single and double mutant phenotypes, these phenotypes may be related. The agravitropic phenotype and abnormal gravi-stimulation response are unique to irk pxc2 double mutants but did not impact growth and maturation of these plants on soil (not shown). These results suggest that enhanced disorganization of root tissues in irk pxc2 may lead to broader defects in overall root growth or that IRK and PXC2 are redundantly required for normal root gravitropism.

\section{PXC2 is not functionally equivalent to IRK}

PXC2 and IRK share $57.1 \%$ identity across the entire protein and $65.1 \%$ identity within their intracellular domains and have overlapping expression and polar accumulation patterns. pxc2 mutants exhibit a subset of the irk mutant phenotypes and the irk pxc2 double mutant phenotype is enhanced suggesting functional redundancy. Some related, functionally redundant LRR-RLKs have also been shown to be functionally equivalent when misexpressed. For example, across the entire protein, ERECTA (ER) is $62-63 \%$ identical to its functional paralogs ERECTA-LIKE 1 (ERL1) and ERL2 and they are functionally equivalent to the ER (Shpak et al., 2004). The same was found for BRI1 and closely related BRI1-LIKE 1 (BRL1) and BRL3, which are $49 \%$ identical to BRI1 (Caño-Delgado et al., 2004). These proteins have comparable amino acid identity as PXC2 and IRK have to each other. Therefore, to further investigate the functional relationship between PXC2 and IRK, we examined whether PXC2 was functionally equivalent to IRK.

pSCR-driven misexpression of IRK-GFP was shown to be sufficient to rescue the irk-4 phenotype, including the increase in endodermal LADs and stele area $60 \mu \mathrm{m}$ above the QC (Campos et al., 2020). Thus, we wanted to determine if $p S C R$ driven expression of PXC2-GFP could similarly rescue the irk-4 phenotype. In young seedlings (4dps), expression of pSCR:PXC2:GFP in irk-4 rescued the excess endodermal LAD phenotype and while stele area was significantly reduced compared to irk-4, it was not fully rescued (Figure 4A, B). Just two days later ( $6 \mathrm{dps}$ ), we found only partial rescue of both the endodermal LAD and stele area phenotypes (Figure 4C, D). These observations were similar whether the transgene was segregating or was homozygous in irk-4. This suggests that in irk-4 roots expressing pSCR:PXC2:GFP increased stele area precedes excess endodermal LADs. Overall, our results indicate that PXC2 is not functionally equivalent to and cannot fully compensate for the loss of IRK.

\section{DISCUSSION}

With the characterization of PXC2, we have identified another LRR-RLK involved in polarized cell-cell communication and a new player regulating cell divisions that contribute to root patterning, particularly in the root's radial axis. Under its own promoter, we show that PXC2-GFP is weakly expressed throughout the root with stronger expression in the LRC, where 
it localizes to the inner polar domain, and in xylem cell types, where it appears nonpolar. Similar to IRK, misexpression of PXC2-GFP reveals polar accumulation in various cell types and accumulation to distinct polar domains in different cell types suggests localization of IRK and PXC2 is informed by adjacent cells and not organ-level or global polarity cues as proposed for polarly localized nutrient transporters (Alassimone et al., 2010; Takano et al., 2010; Barberon et al., 2014) and the SOSEKI proteins (Yoshida et al., 2019), respectively. Thus, we predict that IRK and PXC2 may share common polarization mechanisms in different cell types.

Examination of pxc2 mutant roots revealed increased stele area compared to wild type. This phenotype is mild and observed only when seedlings were grown on media containing a lower concentration of MS salts than our standard growth media. This suggests the consequences of loss of PXC2 function are exacerbated by lower nutrient conditions or more rapid root growth. Because IRK and PXC2 are so closely related and the irk root phenotypes are enhanced in irk pxc2 double mutants, we conclude that PXC2 and IRK act redundantly to inhibit endodermal LADs and restrict stele area in the root meristem (Figure 5A, B). Given the mild abnormal pxc2 phenotype, our data are consistent with a predominant role for IRK in root development (Figure 5B). In wild type, PXC2 and IRK function to limit stele area and repress endodermal LADs, such that there are typically only 8 endodermal cells around the stele (Figure $5 \mathrm{~B}$, left). In pxc2 mutants, we observe an increase in stele area while endodermal cell number remains the same as wild type. This indicates that IRK activity alone is sufficient to repress endodermal LADs, but not to fully restrict stele area (Figure 5B, center-left). In contrast, in irk mutants, increased stele area and endodermal cell number around the stele are observed, indicating PXC2 alone is not sufficient to restrict endodermal LADs and stele area to WT levels (Figure 5B, center-right). In the double mutant, enhanced increases in stele area and endodermal LADs indicate PXC2 and IRK function redundantly to restrict them (Figure 5B, right); additionally, IRK appears fully redundant to PXC2 in repression of endodermal LADs. However, PXC2 and IRK may not be fully redundant with regard to repression of stele area, as it is enlarged in each single mutant. Alternatively, a lower dosage of these gene products may lead to weaker single mutant phenotypes compared to the double mutant.

Although we propose they are redundant, misexpression of PXC2 is not sufficient to compensate for the loss of IRK. We unexpectedly observed that endodermal-specific expression of PXC2-GFP only rescued the endodermal LAD phenotype in irk-4 roots. This is in contrast to $p S C R$ driven expression of IRK-GFP, which rescues the increased endodermal LADs and stele area phenotypes in irk-4. Based on this, we previously concluded that increased stele area was a secondary defect in irk-4 due to excess endodermal LADs. However, the relationship between endodermal cell number and stele area may be more complicated. For example, in pxc2 roots (at $6 \mathrm{dps}$ ) and irk-4 pSCR:PXC2:GFP roots (at $4 \mathrm{dps}$ ), we do not observe excess endodermal LADs; yet stele area is enlarged, suggesting an increase in stele area precedes the occurrence of endodermal LADs in these genotypes. This is supported by the observation that irk-4 pSCR:PXC2:GFP roots at a later time (6 dps) show an enlarged stele and excess endodermal LADs (Figure 4C, D). Thus, it is possible that dysregulation or uncoupling of communication between the endodermis and stele leads to the observed phenotypes, hinting at mechanical feedback between tissues in the root's radial axis. For instance, if excess 
endodermal LADs broaden the root in the radial axis, stele area may increase as a consequence. On the other hand, if stele area increases, endodermal LADs may occur to accommodate this. Further exploration of the cell type-specific functions of IRK and PXC2 during root development will allow deeper functional insights into these proteins, but importantly, can serve as novel tools to dissect the interaction between endogenous genetic control and exogenous mechanical feedback during developmental patterning.

Finally, irk pxc2 double mutants exhibit an agravitropic root growth phenotype, not observed in either single mutant. This suggests that IRK and PXC2 are redundantly required for normal root growth. This growth phenotype may be attributed to the large increases in endodermal cell number and stele area in the double mutant, as directional growth requires the coordinated elongation of neighboring cell types (Sablowski, 2016; Vaahtera et al., 2019). For instance, because the endodermal LADs in the mutants tend to occur nonuniformly in the radial axis (Figures 2F and S4B, (Campos et al. 2020)), regions with smaller, more numerous endodermal cells could impede coordinated cell expansion. Additionally, the increase in endodermal periclinal cell divisions would result in variable numbers of ground tissue layers in the root's radial axis. Thus, difficulties in coordination of cell elongation across root cell layers that are non-uniform in cell number may give rise to an agravitropic growth phenotype. Alternatively, recent data indicates PXC2/CANAR binds to and coordinates the polarization of PIN1, an auxin efflux carrier, in shoot tissues (Hajný et al., 2020) suggesting another explanation for the irk pxc2 root growth phenotype. Root gravitropism requires the (re)polarization of PINs to achieve differential auxin distribution and cell elongation (Friml et al., 2002; Kleine-Vehn et al., 2010). Therefore, if PXC2 and, by extension, closely related IRK, have similar interactions with PINs in the root, this could explain the agravitropic phenotype of the pxc2 irk double mutant and will be an important avenue of future work. Given our collective results, we hypothesize a crucial relationship exists between the polarity and function of PXC2 and IRK and coordinated cell division, radial patterning, and cell elongation during root growth and development.

\section{EXPERIMENTAL PROCEDURES}

\section{Plant Materials and Growth Conditions}

Seeds were surface sterilized with chlorine gas, then plated on media $(\mathrm{pH} 5.7)$ containing 1\% (BD Difco ${ }^{\mathrm{TM}}$ ) Agar and 0.5g/L MES (EMD), supplemented with 1\% sucrose w/v and 1x Murashige and Skoog (MS, Caisson labs) basal salts (our standard growth medium) or $0.2 x \mathrm{MS}$ salts (as noted). Plates were sealed with parafilm or $3 \mathrm{M}$ micropore tape as indicated below. Seeds were stratified on plates in the dark at $4 \mathrm{C}$ for $48-72$ hours and then placed vertically in a Percival growth chamber, with $16 \mathrm{~h}$ light/8h dark cycle at a constant temperature of $22^{\circ} \mathrm{C}$.

\section{Confocal microscopy and analysis of fluorescent reporters}

Roots were stained with $\sim 10 \mu \mathrm{M}$ propidium iodide $(\mathrm{PI})$ solubilized in water for 1-2 min and visualized via laser scanning confocal microscopy on a Leica SP8 upright microscope 
housed in the Van Norman lab. Root meristems were visualized in the median longitudinal, transverse planes, or as Z-stacks. Fluorescence signals were visualized as follows: GFP (excitation $488 \mathrm{~nm}$, emission 492-530 nm), YFP (excitation $514 \mathrm{~nm}$, emission 515-550 nm), and PI (excitation $536 \mathrm{~nm}$, emission 585-660 nm). All confocal images are either median longitudinal optical sections, transverse optical sections, or part of a Z-stack acquired in the root meristematic or elongation zone. Images of roots expressing PXC2 transcriptional or translational fusions were taken at either 5 or $7 \mathrm{dps}$ with no observable differences due to age. Roots misexpressing PXC2-FP using cell type-specific promoters were imaged at $5 \mathrm{dps}$.

\section{Endodermal LAD quantification and stele area measurement}

All images of roots for analysis of endodermal LADs and stele area were taken as Zstacks at $512 \times 512$ resolution with $1 \mu \mathrm{m}$ between scans, speed setting at 600 with a line average of six. Z-compensation was used by increasing 588nm laser intensity while focusing through the $Z$ plane, from $2 \%$ up to $40 \%$ (depending on staining) in order to visualize cell layers adjacent to the slide (in the radial axis).

Total number of endodermal LADs were determined by counting the number of endodermal cells per ring of cells in the transverse plane starting from just above the QC. Because cells of the cortex and endodermis are formed as a pair through periclinal division of a single initial cell, endodermal LADs typically result in two or more small endodermal cells adjacent to a single cortex cell. For each individual root, 15 endodermal rings were scored for occurances of LADs, the presence of an additional longitudinal anticlinal wall within a ring of endodermal cells was scored as an LAD and then summed. If any of the first 15 endodermal cells were damaged (PI infiltration) the root was not used in the analysis.

Stele area was measured in the transverse section located $60 \mu \mathrm{m}$ above the QC using ImageJ software. The inner cell wall of the endodermis was traced and used to calculate the area of the region in ImageJ. If the stele was damaged or staining was too weak to accurately determine the endodermal border then that root was excluded from the analysis.

\section{Phenotypic characterization of pxc2 and irk-4 pxc2 mutants}

pxc2-3/canar-3 (SM_3_31635) and pxc2-1/canar-1 (Salk_055351) seeds were obtained through the Arabidopsis Biological Research Center and genotyped using primers listed in Table S1 to identify homozygous mutants. Age matched seeds from plants grown for three generations in the lab were used in these studies. Each mutant was plated with Col-0 on individual plates. Seeds were sown on plates containing $0.2 x \mathrm{MS}$ media and sealed with paraflim. At $6 \mathrm{dps}$, Z-stacks were obtained for pxc2 mutants and Col-0 root tips ( $\mathrm{n}=10-15$ per genotype) for quantification of endodermal LADs and stele area. No additional root cellular morphology phenotype was observed for either pxc2 mutant.

Standard genetic crosses between pxc2-3 and irk-4 plants were completed to generate irk-4 pxc2-3. F2 plants were genotyped to identify genotypes of interest and then confirmed in subsequent generations. Double mutant seeds collected from genotyped F3s were used in these studies. irk-4 pxc2-3 mutants were plated with irk-4 as the controls on plates containing $1 x$ 
MS media and sealed with parafilm. At 4 dps, Z-stacks were obtained for irk-4 pxc2-3 and irk-4 ( $n=10-15$ per genotype) for quantification of endodermal LADs and stele area. Additional imaging was conducted at $6 \mathrm{dps}$, however, quantification of stele area and endodermal LAD was not performed due to severe defects.

For the root length, straightness ratio, and gravi-stimulation analyses, Col-0, pxc2-3, irk4, and irk-4 pxc2-3 seeds were plated on our standard media and sealed with micropore tape and plates were scanned (EPSON V600) at $7 \mathrm{dps}$. After scanning, the plates were turned $90^{\circ}$ and placed back into the growth chamber for 8 hours, then scanned again. From the first scans, total root length was measured by tracing individual roots from the base of the hypocotyl to the root tip using the segmented line feature in ImageJ. Then, a straight line was drawn from the base of the hypocotyl to the root tip and measured as Dy (Figure 4A). The straightness ratio is calculated by dividing Dy by total root length where a ratio of 1.0 indicates root growth always parallel with the gravity vector. To assess Dx (Figure 4A), a straight line was first drawn directly downward from the base of the hypocotyl to the region parallel to the root tip. Then, a straight line was drawn from this point to the root tip, resulting in the Dx measurement, with a positive value assigned to the rightward direction of root drift as observed in Col-0. From the second scans ( 8 hours after gravistimulation), the angle between $1 \mathrm{~mm}$ of the root tip and the new gravity vector were measured in ImageJ to assess the root's response following gravistimulation.

\section{PXC2-GFP rescue of irk-4}

irk-4 plants homozygous for $p S C R: P X C 2: G F P$, Col-0, and irk-4 mutants were plated on 1x MS media and imaged at $4 \mathrm{dps}$ as previously described. irk-4 mutants segregating for pSCR:PXC2:GFP and Col-0 were plated together on media previously described and imaged at 6 dps. Two independent transgenic lines (\#4 and \#6) were crossed with irk-4 for analysis at both 4 and 6 dps with similar results.

\section{Vector Construction and Plant Transformation}

Transcriptional and translational reporters were constructed by standard molecular biology methods and utilizing Invitrogen Multisite Gateway ${ }^{\circledR}$ technology (Carlsbad, USA). The $4.7 \mathrm{~kb}$ region upstream of the PXC2 (At5g01890) start codon was amplified from Col-0 genomic DNA and recombined into the Invitrogen $\mathrm{pENTR}{ }^{\mathrm{TM}} 5$ '-TOPO ${ }^{\circ}$ TA vector as the promoter of $P X C 2$. For the transcriptional reporter, $p P X C 2$ drove endoplasmic reticulum-localized green fluorescent protein (erGFP) as previously described (Van Norman et al., 2014). For translational fusions, the genomic fragment encoding PXC2 from the ATG up to, but excluding the stop codon (including introns, $3.0 \mathrm{~kb}$ ), was amplified from Col-0 genomic DNA and recombined into the Invitrogen pENTR ${ }^{T M}$ DIRECTIONAL TOPO® (pENTR-D-TOPO) vector and fused to a Cterminal GFP tag as previously described (Van Norman et al., 2014). Specific primers for PXC2 cloning are listed in Table S1.

Cell type- or layer-specific promoters ( $p S C R_{2.0}, p \mathrm{CO}_{2}$, and $\left.p W E R\right)$ as previously described (Campos et al., 2020; Lee et al., 2006), and pSMB as described (Bennett et al., 2010) were used to drive PXC2-GFP. The various Gateway compatible fragments were recombined 
together with the dpGreen-BarT (Lee et al., 2006) or dpGreen-NorfT (Norflurazon resistant) destination vectors. The dpGreenNorfT was generated by combining the backbone of dpGreenBarT with the p35S::tpCRT1 and terminator insert from $p G / 10125$. Within the target region of the dpGreenBarT, one Acll site was mutated with the QuickChangeXL kit (Stratagene) and primers as in Table S1. Plasmids were amplified in ccdB-resistant $E$. coli and plasmids prepped with a Bio Basic Plasmid DNA Miniprep kit. 34uL of the modified dpGreenBarT and unmodified $p G / 10125$ were digested with $1 \mathrm{ul}$ each Fspl and Acll in CutSmart buffer (NEB) for $1 \mathrm{hr}$ at $37^{\circ} \mathrm{C}$. Digests were subjected to gel electrophoresis on a $1 \%$ agarose gel. The $5866 \mathrm{bp}$ fragment from the dpGreenBarT and 2592bp fragment from the $p G I I I 0125$ were extracted with a Qiagen MinElute Gel Extraction kit. The fragments were then ligated at 1:1 volumetric ratio (20ng vector; 8.8ng insert) using T4 DNA ligase incubated at $16^{\circ} \mathrm{C}$ overnight before transformation into ccdB-resistant $E$. coli.

Expression vectors were then transformed into Agrobacterium strain GV3101 (Koncz et al., 1992) and then into Col-0 plants by the floral dip method (Clough and Bent, 1998).

Transformants were identified using standard methods. For each reporter, T2 lines with a 3:1 ratio of resistant:sensitive seedlings, indicating the transgene is inherited as a single locus, were selected for propagation. These T2 plants were allowed to self and among the subsequent T3 progeny, those with $100 \%$ resistant seedlings, indicating that the transgene was homozygous, were used in further analyses. For each reporter, at least three independent lines with the similar relative expression levels and localization patterns were selected for imaging by confocal microscopy.

\section{Figure construction}

Confocal images for stele area measurement and endodermal LAD quantification were examined in ImageJ (http://imagej.nih.gov/ij) (Schneider et al., 2012). For use in figures, raw confocal images were converted to .TIF format using Leica software (LASX) and were assembled in Adobe Photoshop. Statistical analysis and graphical representation of all data in this publication was done using PRISM8 (GraphPad Software, https://www.graphpad.com/, San Diego, USA). Type of graphs and statistical analysis used are listed in figure legends. Schematics were created in Illustrator and then figures containing images, graphs, and schematics were assembled in Adobe Illustrator.

\section{qRT-PCR analysis}

Total RNA was isolated from seedlings at 7dps of three independent biological replicates for each Col-0 (wild type), SM_3_31635 (pxc2-3/canar-3) and Salk_055351 (pxc2-1/canar-1) using Qiagen RNeasy Plant Mini Kit. Seeds of all three genotypes were sown together on plates containing 1x MS media, sealed with parafilm, and stratified in the dark at $4^{\circ} \mathrm{C}$ for 3 overnights. Following total RNA extraction, samples were examined for concentration and purity and stored at $-80^{\circ} \mathrm{C}$ prior to cDNA synthesis. For cDNA synthesis (RevertAid First Strand cDNA Synthesis Kit, Thermo Scientific), $1 \mu \mathrm{g}$ of total RNA was used to normalize for varying RNA concentrations within biological samples, and the Oligo(dT)18 primer was used to generate cDNA. To examine PXC2 expression in irk-4, previously obtained RNA samples (Campos et al. 2020) were used. qRT-PCR reactions were done using IQ SYBR Green Supermix (BioRad) and analysis was 
performed on the CFX Connect Real-Time System housed in the Integrative Institute of Genome Biology Genomics Core facility at UC-Riverside. The reaction conditions for all primer pairs were: $95^{\circ} \mathrm{C}$ for $3 \mathrm{~min}$, followed by 40 cycles of $95^{\circ} \mathrm{C}$ for 10 s and $60^{\circ} \mathrm{C}$ for 30 s. Primer pair efficiency was calculated for each reaction using standard curve data. For each genotype and biological replicate, three technical replicates were performed, and all transcript levels were normalized to PHOSPHATASE 2A (PP2A) (Czechowski et al., 2005). Data analysis was done using Bio-Rad CFX Maestro 1.1 (version 4.1.2433.1219).

\section{Gene Structure and amino acid sequence comparisons}

DNA sequence of PXC2 including 5' and 3' UTR was copied into the web-based

http://wormweb.org/exonintron tool and annotated with the positions of the T-DNA insertions based on DNA sequencing for pxc2-3 and using publicly available data from The Arabidopsis Information Resource (TAIR, www.arabidopsis.org) for pxc2-1/canar-1 and pxc2-2/canar-2. Percent identity for PXC2 and IRK and of ERL1 and ERL2 to ER and BRL1 and BRL3 to BRI1 were obtained using the web based Clustal Omega tool (https://www.ebi.ac.uk/Tools/msa/clustalo/).

\section{ACKNOWLEDGMENTS}

We thank Roya Campos for assistance in the construction of several PXC2-GFP misexpression vectors and Dr. Dawn Nagel, Dr. Cecilia Rodriguez-Furlan, Roya Campos, and Jessica Toth for discussions of the project and feedback on the manuscript while it was in preparation. We also thank Dr. Erin Sparks (University of Delaware) for providing the NorfT Gateway ${ }^{\circledR}$ compatible destination vector. We appreciate access to and assistance from the Institute of Integrative Genome Biology Genomics Core Facility (UC, Riverside) for qRT-PCR experiments. This work was supported funds award to JMVN, specifically by Initial Compliment (IC) funds from the University of California at Riverside, USDA-NIFA-CA-R-BPS-5156-H, and by an NSF CAREER award (\#1751385).

\section{CONFLICTS OF INTEREST}

None declared.

\section{SUPPORTING INFORMATION}

Figure S1: Confocal micrographs showing PXC2 promoter activity ( $P P X C 2$ :erGFP) and PXC2GFP accumulation (driven by $p P X C 2$ ) across the root developmental zones.

Figure S2: Schematic of the $P X C 2$ gene structure and expression analyses of $P X C 2$ and $I R K$ in the pxc2 mutant alleles and expression of $P X C 2$ in irk-4.

Figure S3: Confocal micrographs of Col-0 and pxc2-1/canar-1 at $6 \mathrm{dps}$ and graphs showing similar stele area and endodermal LAD phenotypes in pxc2-3 and pxc2-1/canar-1 roots. 
Figure S4: Confocal micrographs showing increased disorganization of irk-4 pxc2-3 double mutant roots at $6 \mathrm{dps}$, and showing rescue back to the irk-4 phenotype upon expression of pPXC2:PXC2:GFP. Additionally, the pxc2-3 and irk-4 single mutant root growth phenotypes.

Table S1: List of primers used.

\section{AUTHOR CONTRIBUTIONS}

Conceptualization: J.M.V.N.; Methodology and Investigation: J.M.V.N. and J.G.; Resources: J.M.V.N. and J.G.; Writing - Original Draft: J.M.V.N and J.G; Review and Editing: J.M.V.N. and J.G; Visualization: J.G. and J.M.V.N.; Supervision: J.M.V.N.; Funding Acquisition: J.M.V.N.

\section{REFERENCES}

Alassimone, J., Naseer, S., and Geldner, N. (2010). A developmental framework for endodermal differentiation and polarity. Proc. Natl. Acad. Sci. U. S. A. 107: 5214-5219.

Arribas-Hernández, L., Simonini, S., Hansen, M.H., Paredes, E.B., Bressendorff, S., Dong, Y., Østergaard, L., and Brodersen, P. (2020). Recurrent requirement for the m6AECT2/ECT3/ECT4 axis in the control of cell proliferation during plant organogenesis. Development 147.

Barberon, M., Dubeaux, G., Kolb, C., Isono, E., Zelazny, E., and Vert, G. (2014). Polarization of IRON-REGULATED TRANSPORTER 1 (IRT1) to the plant-soil interface plays crucial role in metal homeostasis. Proc. Natl. Acad. Sci. U. S. A. 111: 8293-8298.

Bennett, T., van den Toorn, A., Sanchez-Perez, G.F., Campilho, A., Willemsen, V., Snel, B., and Scheres, B. (2010). SOMBRERO, BEARSKIN1, and BEARSKIN2 regulate root cap maturation in Arabidopsis. Plant Cell 22: 640-654.

van den Berg, C., Willemsen, V., Hendriks, G., Weisbeek, P., and Scheres, B. (1997). Shortrange control of cell differentiation in the Arabidopsis root meristem. Nature 390: 287-289.

van den Burg, C., Willemsen, V., Hage, W., Weisbeek, P., and Scheres, B. (1995). Cell fate in the Arabidopsis root meristem determined by directional signaling. Nature 378: 62.

Camilleri, C., Azimzadeh, J., Pastuglia, M., Bellini, C., Grandjean, O., and Bouchez, D. (2002). The Arabidopsis TONNEAU2 gene encodes a putative novel protein phosphatase $2 \mathrm{~A}$ regulatory subunit essential for the control of the cortical cytoskeleton. Plant Cell 14: 833-845.

Campos, R., Goff, J., Rodriguez-Furlan, C., and Van Norman, J.M. (2020). The Arabidopsis Receptor Kinase IRK Is Polarized and Represses Specific Cell Divisions in Roots. Dev. Cell 52: 183-195.e4.

Caño-Delgado, A., Yin, Y., Yu, C., Vafeados, D., Mora-García, S., Cheng, J.-C., Nam, K.H., Li, J., and Chory, J. (2004). BRL1 and BRL3 are novel brassinosteroid receptors that 
function in vascular differentiation in Arabidopsis. Development 131: 5341-5351.

Chaiwanon, J., Wang, W., Zhu, J.-Y., Oh, E., and Wang, Z.-Y. (2016). Information Integration and Communication in Plant Growth Regulation. Cell 164: 1257-1268.

Clough, S.J. and Bent, A.F. (1998). Floral dip: a simplified method for Agrobacterium-mediated transformation of Arabidopsis thaliana. Plant J. 16: 735-743.

Correll, M.J. and Kiss, J.Z. (2005). The roles of phytochromes in elongation and gravitropism of roots. Plant Cell Physiol. 46: 317-323.

Czechowski, T., Stitt, M., Altmann, T., Udvardi, M.K., and Scheible, W.-R. (2005). GenomeWide Identification and Testing of Superior Reference Genes for Transcript Normalization in Arabidopsis. Plant Physiol. 139: 5-17.

Dietrich, D. et al. (2017). Root hydrotropism is controlled via a cortex-specific growth mechanism. Nat Plants 3: 17057.

Diévart, A. and Clark, S.E. (2003). Using mutant alleles to determine the structure and function of leucine-rich repeat receptor-like kinases. Curr. Opin. Plant Biol. 6: 507-516.

Dolan, L., Janmaat, K., Willemsen, V., Linstead, P., Poethig, S., Roberts, K., and Scheres, B. (1993). Cellular organisation of the Arabidopsis thaliana root. Development 119: 71-84.

Dyson, R.J. et al. (2014). Mechanical modelling quantifies the functional importance of outer tissue layers during root elongation and bending. New Phytol. 202: 1212-1222.

Facette, M.R., Rasmussen, C.G., and Van Norman, J.M. (2018). A plane choice: coordinating timing and orientation of cell division during plant development. Curr. Opin. Plant Biol. 47: 47-55.

Friml, J., Wiśniewska, J., Benková, E., Mendgen, K., and Palme, K. (2002). Lateral relocation of auxin efflux regulator PIN3 mediates tropism in Arabidopsis. Nature 415: 806809.

Grabov, A., Ashley, M.K., Rigas, S., Hatzopoulos, P., Dolan, L., and Vicente-Agullo, F. (2005). Morphometric analysis of root shape. New Phytol. 165: 641-651.

Hajný, J. et al. (2020). Receptor kinase module targets PIN-dependent auxin transport during canalization. Science 370: 550-557.

Hodge, A., Berta, G., Doussan, C., Merchan, F., and Crespi, M. (2009). Plant root growth, architecture and function. Plant Soil 321: 153-187.

Kleine-Vehn, J., Ding, Z., Jones, A.R., Tasaka, M., Morita, M.T., and Friml, J. (2010). Gravity-induced PIN transcytosis for polarization of auxin fluxes in gravity-sensing root cells. Proc. Natl. Acad. Sci. U. S. A. 107: 22344-22349.

Koncz, C., Németh, K., Rédei, G.P., and Schell, J. (1992). T-DNA insertional mutagenesis in Arabidopsis. Plant Mol. Biol. 20: 963-976.

Lee, J.-Y., Colinas, J., Wang, J.Y., Mace, D., Ohler, U., and Benfey, P.N. (2006). Transcriptional and posttranscriptional regulation of transcription factor expression in 
Arabidopsis roots. Proc. Natl. Acad. Sci. U. S. A. 103: 6055-6060.

Lee, M.M. and Schiefelbein, J. (1999). WEREWOLF, a MYB-Related Protein in Arabidopsis, Is a Position-Dependent Regulator of Epidermal Cell Patterning. Cell 99: 473-483.

Marhava, P., Hoermayer, L., Yoshida, S., Marhavý, P., Benková, E., and Friml, J. (2019). Re-activation of Stem Cell Pathways for Pattern Restoration in Plant Wound Healing. Cell 177: 957-969.e13.

Martinez, P., Luo, A., Sylvester, A., and Rasmussen, C.G. (2017). Proper division plane orientation and mitotic progression together allow normal growth of maize. Proc. Natl. Acad. Sci. U. S. A. 114: 2759-2764.

Meyerowitz, E.M. (1997). Genetic control of cell division patterns in developing plants. Cell 88: 299-308.

Paquette, A.J. and Benfey, P.N. (2005). Maturation of the ground tissue of the root is regulated by gibberellin and SCARECROW and requires SHORT-ROOT. Plant Physiol. 138: 636640.

Rasmussen, C.G. and Bellinger, M. (2018). An overview of plant division-plane orientation. New Phytol. 219: 505-512.

Sabatini, S., Heidstra, R., Wildwater, M., and Scheres, B. (2003). SCARECROW is involved in positioning the stem cell niche in the Arabidopsis root meristem. Genes Dev. 17: 354358.

Sablowski, R. (2016). Coordination of plant cell growth and division: collective control or mutual agreement? Curr. Opin. Plant Biol. 34: 54-60.

Scheres, B. and Benfey, P.N. (1999). ASYMMETRIC CELL DIVISION IN PLANTS. Annu. Rev. Plant Physiol. Plant Mol. Biol. 50: 505-537.

Schneider, C.A., Rasband, W.S., and Eliceiri, K.W. (2012). NIH Image to ImageJ: 25 years of image analysis. Nat. Methods 9: 671-675.

Shao, W. and Dong, J. (2016). Polarity in plant asymmetric cell division: Division orientation and cell fate differentiation. Dev. Biol. 419: 121-131.

Shiu, S.H. and Bleecker, A.B. (2003). Expansion of the receptor-like kinase/Pelle gene family and receptor-like proteins in Arabidopsis. Plant Physiol. 132: 530-543.

Shiu, S.H. and Bleecker, A.B. (2001). Receptor-like kinases from Arabidopsis form a monophyletic gene family related to animal receptor kinases. Proc. Natl. Acad. Sci. U. S. A. 98: 10763-10768.

Shpak, E.D., Berthiaume, C.T., Hill, E.J., and Torii, K.U. (2004). Synergistic interaction of three ERECTA-family receptor-like kinases controls Arabidopsis organ growth and flower development by promoting cell proliferation. Development 131: 1491-1501.

Su, S.-H., Gibbs, N.M., Jancewicz, A.L., and Masson, P.H. (2017). Molecular Mechanisms of Root Gravitropism. Curr. Biol. 27: R964-R972. 
Takano, J., Tanaka, M., Toyoda, A., Miwa, K., Kasai, K., Fuji, K., Onouchi, H., Naito, S., and Fujiwara, T. (2010). Polar localization and degradation of Arabidopsis boron transporters through distinct trafficking pathways. Proc. Natl. Acad. Sci. U. S. A. 107: 5220-5225.

Vaahtera, L., Schulz, J., and Hamann, T. (2019). Cell wall integrity maintenance during plant development and interaction with the environment. Nat Plants 5: 924-932.

Van Norman, J.M., Breakfield, N.W., and Benfey, P.N. (2011). Intercellular communication during plant development. Plant Cell 23: 855-864.

Van Norman, J.M., Zhang, J., Cazzonelli, C.I., Pogson, B.J., Harrison, P.J., Bugg, T.D.H., Chan, K.X., Thompson, A.J., and Benfey, P.N. (2014). Periodic root branching in Arabidopsis requires synthesis of an uncharacterized carotenoid derivative. Proc. Natl. Acad. Sci. U. S. A. 111: E1300-9.

Wang, J., Kucukoglu, M., Zhang, L., Chen, P., Decker, D., Nilsson, O., Jones, B., Sandberg, G., and Zheng, B. (2013). The Arabidopsis LRR-RLK, PXC1, is a regulator of secondary wall formation correlated with the TDIF-PXY/TDR-WOX4 signaling pathway. BMC Plant Biol. 13: 94.

Willemsen, V., Bauch, M., Bennett, T., Campilho, A., Wolkenfelt, H., Xu, J., Haseloff, J., and Scheres, B. (2008). The NAC domain transcription factors FEZ and SOMBRERO control the orientation of cell division plane in Arabidopsis root stem cells. Dev. Cell 15: 913-922.

Wu, S., O’Lexy, R., Xu, M., Sang, Y., Chen, X., Yu, Q., and Gallagher, K.L. (2016). Symplastic signaling instructs cell division, cell expansion, and cell polarity in the ground tissue of Arabidopsis thaliana roots. Proc. Natl. Acad. Sci. U. S. A. 113: 11621-11626.

Wysocka-Diller, J.W., Helariutta, Y., Fukaki, H., Malamy, J.E., and Benfey, P.N. (2000). Molecular analysis of SCARECROW function reveals a radial patterning mechanism common to root and shoot. Development 127: 595-603.

Yoshida, S., van der Schuren, A., van Dop, M., van Galen, L., Saiga, S., Adibi, M., Möller, B., Ten Hove, C.A., Marhavy, P., Smith, R., Friml, J., and Weijers, D. (2019). A SOSEKIbased coordinate system interprets global polarity cues in Arabidopsis. Nat Plants 5: 160166.

\section{FIGURE LEGENDS}

Figure 1. PXC2 is polarly localized in root cell types. (A) Schematics of an Arabidopsis thaliana root meristem with transverse (left) and longitudinal (right) sections showing cell types and their organization (adapted from (Campos et al., 2020)). (B-G) Confocal micrographs of Arabidopsis root meristems stained with propidium iodide (PI, magenta) to visualize individual cells and showing FP (fluorescent protein) localization (green) with PI + FP (left) and FP alone (right). (B) PXC2-GFP localization when driven by $p P X C 2$. (C-F) Polar localization of PXC2-FP upon misexpression by (C) $p S M B$, (D) $p W E R,(E) p C O 2$, and $(F) p S C R$. $(G)$ Schematic summary of PXC2-FP localization in 
root cell types. Cell type abbreviations as indicated in (A). Scale bars: $25 \mu \mathrm{m}$.

Figure 2. Abnormal pxc2 phenotype and enhanced phenotype of irk pxc2 double mutant. $(A, B, E, F)$ Confocal micrographs of Arabidopsis root meristems stained with $\mathrm{PI}$ (magenta). (A, B) Transverse optical sections of Col-0 and pxc2-3 roots at 6 days post stratification (dps). (C, D) Graphs showing total endodermal LADs and stele area in Col-0 and pxc2-3 at 6 dps ( $n=12$ roots per genotype). (E, F) Transverse optical sections of irk-4 and irk-4 pxc2-3 roots at 4 dps. (G, H) Graphs showing endodermal LADs and stele area in irk pxc2 double mutants compared to irk single mutants (irk-4 n $=9$, irk-4 pxc2-3 $n=11$ ). Data shown are from a single representative biological replicate of $\geq 3$, all with similar results. Scatter plots $(C, G)$ : Total number of LADs in individual roots represented by colored symbols with the black bar indicating the mean for each data set. Box plots $(D, H)$ : whiskers indicate min/max with interquartile range and mean shown in colored boxes/lines, respectively, and black dots indicate measurements for individual roots. In the micrographs $(A, B, E, F)$, endodermal cells are shaded, cyan arrowheads indicate LADs, and yellow arrowheads indicate periclinal divisions. Scale bar: $25 \mu \mathrm{m}$. Abbreviations: $\mathrm{E}$ - endodermis, $\mathrm{C}$ - cortex, $\mathrm{x}-\mathrm{Xylem}$, ns = not significant, ${ }^{* *}=p$ value $<0.01,{ }^{* * *}=p$ value $<0.001,{ }^{* * *}=p$ value $<0.0001$, Student's t-test.

Figure 3. irk pxc2 roots exhibit abnormal growth phenotypes. (A) Schematics of seedlings with different growth phenotypes with measurements indicated for calculating straightness ratio and Dx distance. (B) Col-0 and (C) irk-4 pxc2-3 seedlings plated on standard media and sealed with tape at $7 \mathrm{dps}$. Scale bar $=10 \mathrm{~mm}$. Graphs of root growth and phenotypes in Col-0 and irk-4 pxc2-3 roots at $7 \mathrm{dps}(\mathrm{D})$ Total root length, (E) straightness ratio and (G-I) graphs of Dx distance in (G) Col-0 and pxc2-3 ( $\mathrm{n}=15$ per genotype), $(\mathrm{H})$ Col-0 and irk-4 ( $\mathrm{n}=15$ per genotype), Col-0 and irk-4 pxc2-3 (Col-0 $\mathrm{n}=$ 14 , irk-4 pxc2-3 n = 13). (F) Root tip angle of Col-0 (top) and irk-4 pxc2-3 (bottom) roots $8 \mathrm{~h}$ after gravistimulation by turning plates $90^{\circ}$. Colored lines $=$ angle of individual root tips. Col-0 $(n=14)$, irk-4 pxc2-3 $(n=13)$. New gravity vector $\left(90^{\circ}\right)$ indicated by $g$ and black arrow, previous gravity vector aligned with $0^{\circ}$. Scatter plots (E, G-I): Data for individual roots indicated by colored symbols with the black bar indicating the mean for each data set. Error bars = standard deviation. All data shown is from a single biological replicate of $\geq 3$. n.s $=$ not significant, ${ }^{* *}=p$ value $<0.01,{ }^{* * *}=p$ value $<0.001,{ }^{* * *}=p$ value $<0.0001$, Student's t-test.

Figure 4. PXC2 partially rescues abnormal root phenotypes in irk. Graphs showing total number of LADs and stele area in (A, B) Col-0, irk-4 pSCR:PXC2:GFP, and irk-4 plants (at $4 \mathrm{dps}, 9-10$ roots per genotype) and (C, D) Col-0 and irk-4 plants segregating for $p S C R: P X C 2: G F P$ (at $6 \mathrm{dps}$, Col-0 $\mathrm{n}=10$, GFP $+\mathrm{n}=10, \mathrm{GFP}-\mathrm{n}=6-7$ ). All data 
shown are from a single, representative biological replicate of $\geq 3$. Scatter plots $(A, C)$ :

Total number of LADs in individual roots indicated by colored symbols with the black bar indicating the mean for each data set. Box plots $(B, D)$ : whiskers indicate $\mathrm{min} / \mathrm{max}$ with interquartile range and mean shown in colored boxes/lines, respectively, and black dots indicate measurements for individual roots. Abbreviations: $n s=$ not significant, ${ }^{*}=p$ value $<0.05,{ }^{* *}=p$ value $<0.01,{ }^{* * *}=p$ value $<0.001,{ }^{* * * *}=p$ value $<0.0001$, Student's t-test.

\section{Figure 5. Summary IRK and PXC2 function to restrict stele area and endodermal} LADs and phenotypic outcomes. (A) Schematic representations of transverse sections of the root showing phenotypes observed in endodermal cells and stele in Col$0, p x c 2$, irk, and irk pxc2. Highlighted endodermal cells indicate the occurrence of LADs. (B) Diagrams illustrating the relative roles of IRK and PXC2 in each genotype with regard to restriction of stele area and endodermal LADs. Weight of the lines indicates the proposed contribution of each protein to the processes with the phenotypic outcomes listed below each. 


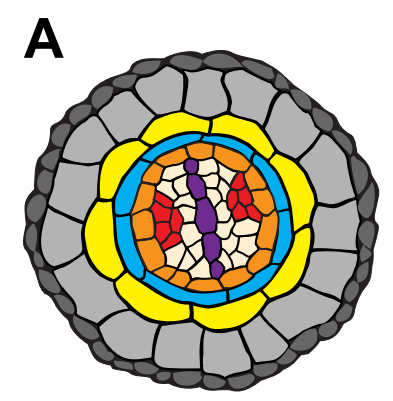

$\square$ Quiescent center (QC) $\square$ Cortex/Endodermal initial (CEI) $\square$ CEl daughter (CEID) $\square$ Cortex (C) $\square$ Endodermis $(\mathrm{E})$ $\square$ Pericycle (P) $\square$ Vascular cambium
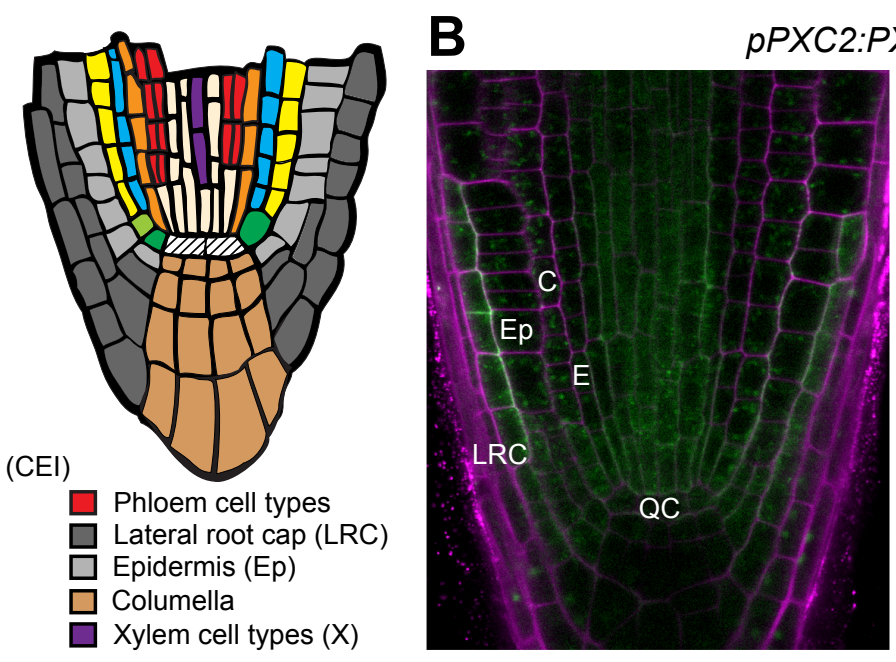

pPXC2:PXC2:GFP
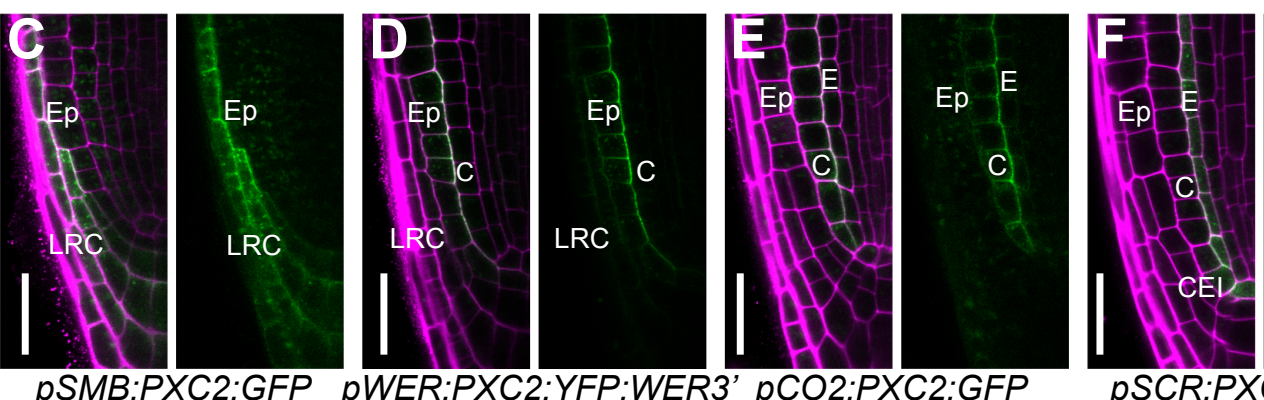

pSCR:PXC2:GFP
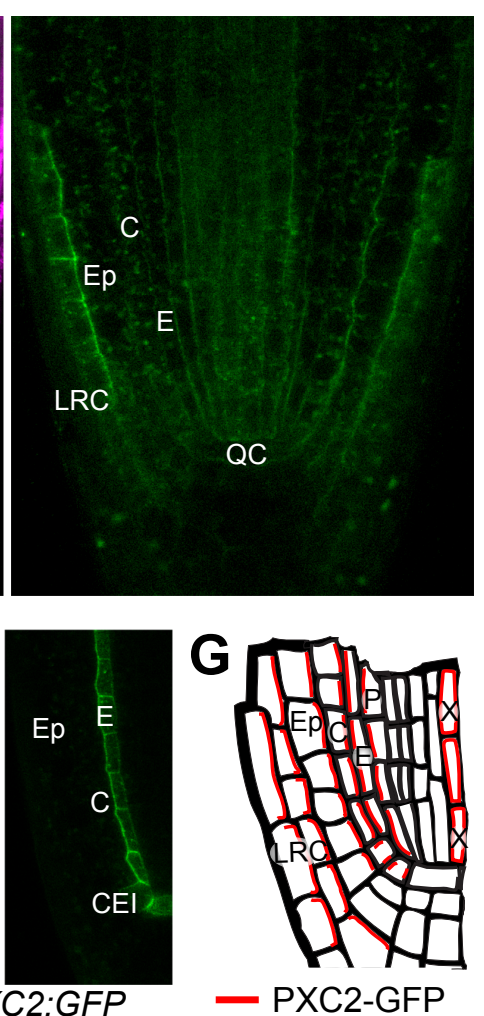

Figure 1. PXC2 is polarly localized in root cell types. (A) Schematics of an Arabidopsis thaliana root meristem with transverse (left) and longitudinal (right) sections showing cell types and their organization (adapted from (Campos et al., 2020)). (B-G) Confocal micrographs of Arabidopsis root meristems stained with propidium iodide (PI, magenta) to visualize individual cells and showing FP (fluorescent protein) localization (green) with PI + FP (left) and FP alone (right). (B) PXC2-GFP localization when driven by $p P X C 2$. (C-F) Polar localization of PXC2-FP upon misexpression by (C) $p S M B$, (D) pWER, (E) pCO2, and (F) pSCR. (G) Schematic summary of PXC2-FP localization in root cell types. Cell type abbreviations as indicated in (A). Scale bars: $25 \mu \mathrm{m}$. 
Figure 2
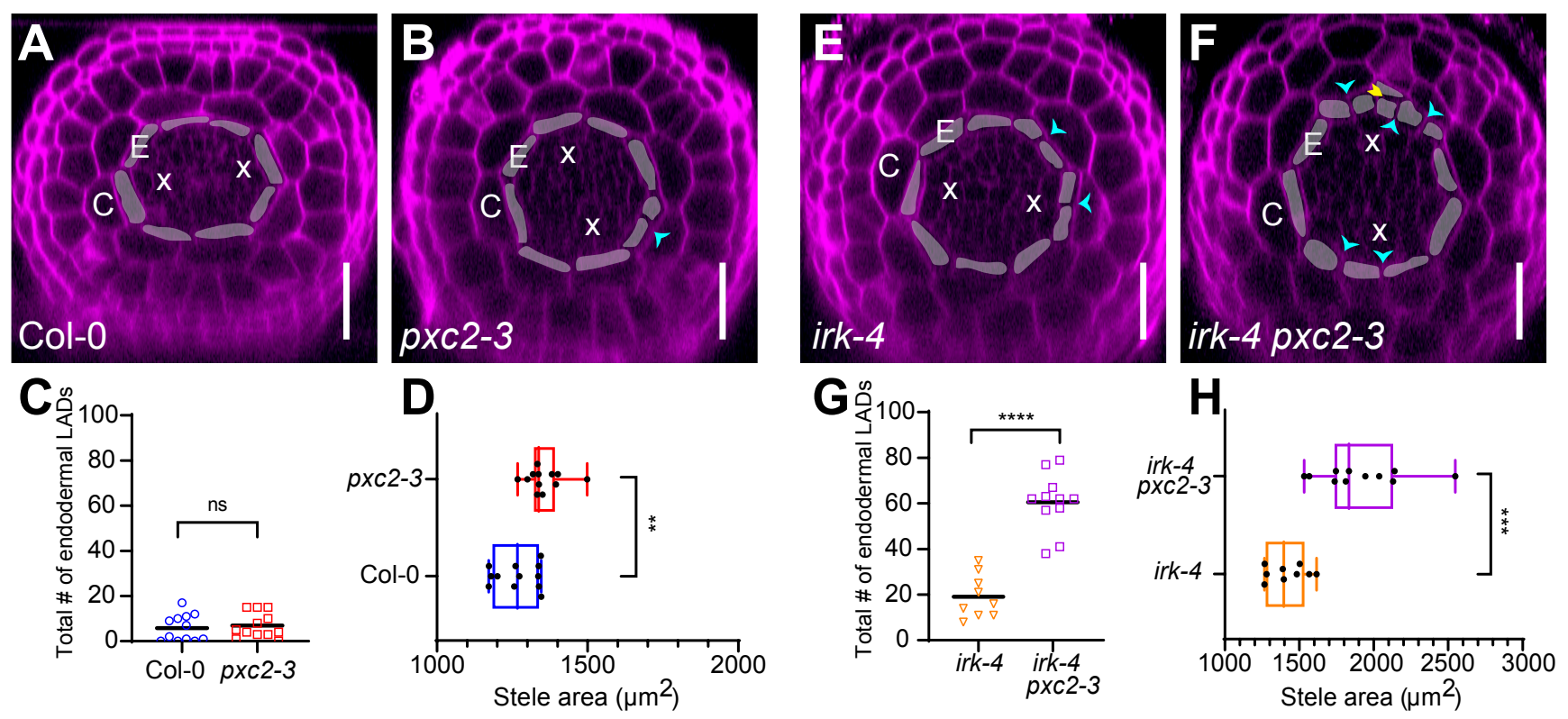

Figure 2. Abnormal pxc2 phenotype and enhanced phenotype of irk pxc2 double mutant. (A, B, E, F) Confocal micrographs of Arabidopsis root meristems stained with PI (magenta). (A, B) Transverse optical sections of Col-0 and pxc2-3 roots at 6 days post stratification (dps). (C, D) Graphs showing total endodermal LADs and stele area in Col-0 and $p x c 2-3$ at 6 dps $(n=12$ roots per genotype). (E, F) Transverse optical sections of irk-4 and irk-4 pxc2-3 roots at $4 \mathrm{dps}$. (G, H) Graphs showing endodermal LADs and stele area in irk pxc2 double mutants compared to irk single mutants (irk-4 $n=9$, irk-4 pxc2-3 $n=11$ ). Data shown are from a single representative biological replicate of $\geq 3$, all with similar results. Scatter plots $(C, G)$ : Total number of LADs in individual roots represented by colored symbols with the black bar indicating the mean for each data set. Box plots $(\mathrm{D}, \mathrm{H})$ : whiskers indicate $\mathrm{min} / \mathrm{max}$ with interquartile range and mean shown in colored boxes/lines, respectively, and black dots indicate measurements for individual roots. In the micrographs (A, $B, E$, $F$ ), endodermal cells are shaded, cyan arrowheads indicate LADs, and yellow arrowheads indicate periclinal divisions. Scale bar: $25 \mu \mathrm{m}$. Abbreviations: $\mathrm{E}$ - endodermis, $\mathrm{C}-$ cortex, $\mathrm{x}-\mathrm{Xylem}$, ns $=$ not significant, ${ }^{* *}=p$ value $<0.01,{ }^{* *}=p$ value $<0.001,{ }^{* * *}=p$ value $<0.0001$, Student's t-test. 
Figure 3
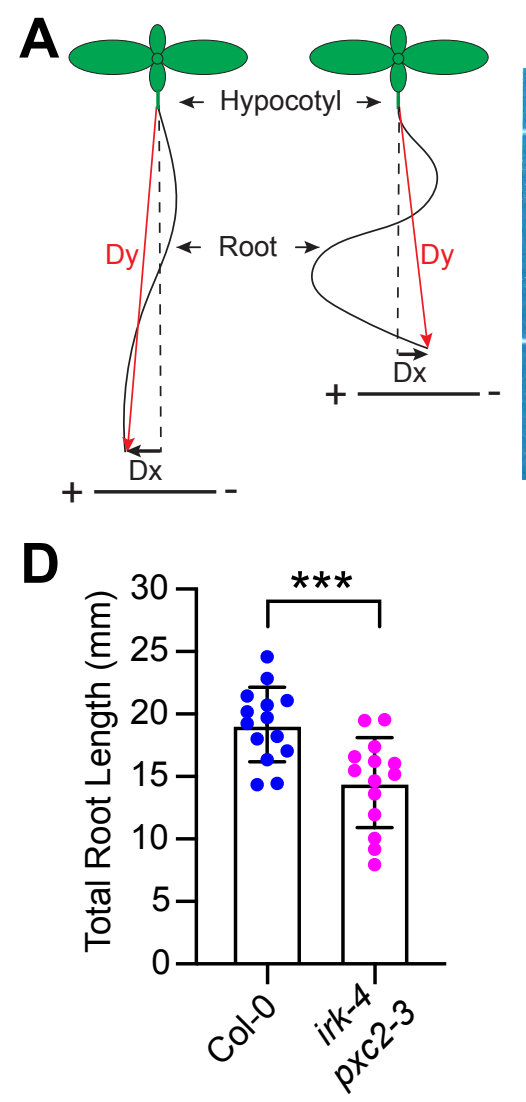

G
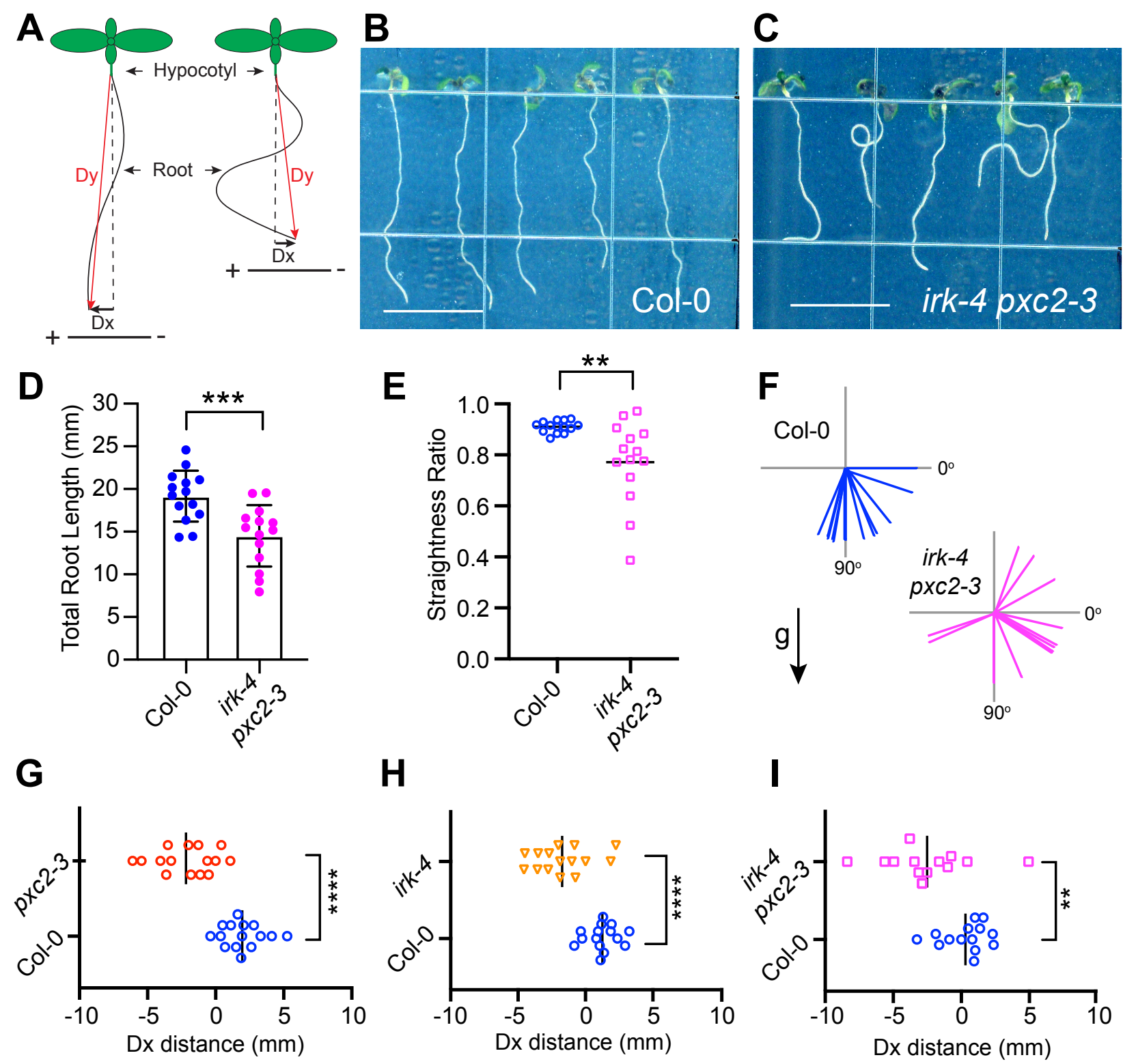

H
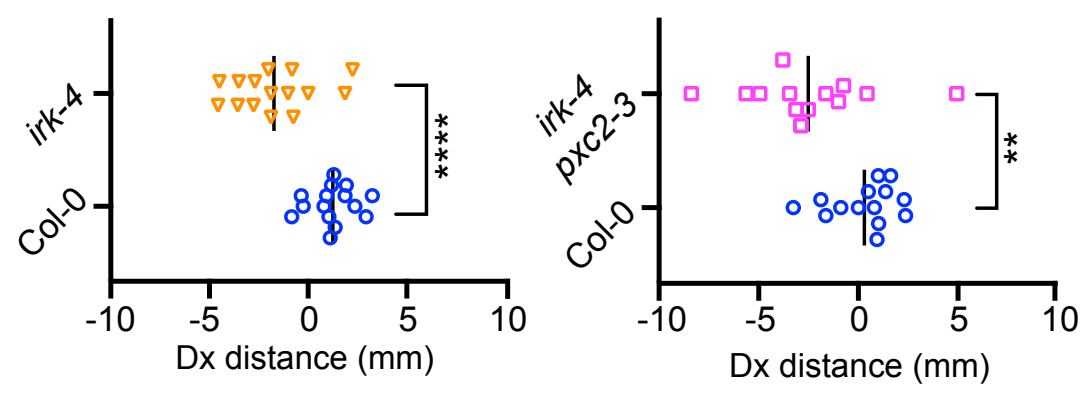

Figure 3. irk pxc2 roots exhibit abnormal growth phenotypes. (A) Schematics of seedlings with different growth phenotypes with measurements indicated for calculating straightness ratio and Dx distance. (B) Col-0 and (C) irk-4 pxc2-3 seedlings plated on standard media and sealed with tape at $7 \mathrm{dps}$. Scale bar $=10 \mathrm{~mm}$. Graphs of root growth and phenotypes in Col-0 and irk-4 pxc2-3 roots at $7 \mathrm{dps}(\mathrm{D})$ Total root length, (E) straightness ratio and (G-I) graphs of Dx distance in (G) Col-0 and pxc2-3 ( $\mathrm{n}=15$ per genotype), $(\mathrm{H})$ Col-0 and $i r k-4(\mathrm{n}=15$ per genotype), Col-0 and irk-4 pxc2-3 (Col-0 $n=14$, irk-4 pxc2-3 $n=13)$. (F) Root tip angle of Col-0 (top) and irk-4 pxc2-3 (bottom) roots $8 \mathrm{~h}$ after gravistimulation by turning plates $90^{\circ}$. Colored lines $=$ angle of individual root tips. Col-0 $(n=14)$, irk-4 pxc2-3 $(n=13)$. New gravity vector $\left(90^{\circ}\right)$ indicated by $g$ and black arrow, previous gravity vector aligned with $0^{\circ}$. Scatter plots (E, G-I): Data for individual roots indicated by colored symbols with the black bar indicating the mean for each data set. Error bars = standard deviation. All data shown is from a single biological replicate of $\geq 3$. n.s $=$ not significant, ${ }^{* *}=p$ value $<0.01,{ }^{* * *}=p$ value $<0.001$, $* * \star *=p$ value $<0.0001$, Student's t-test. 
Figure 4
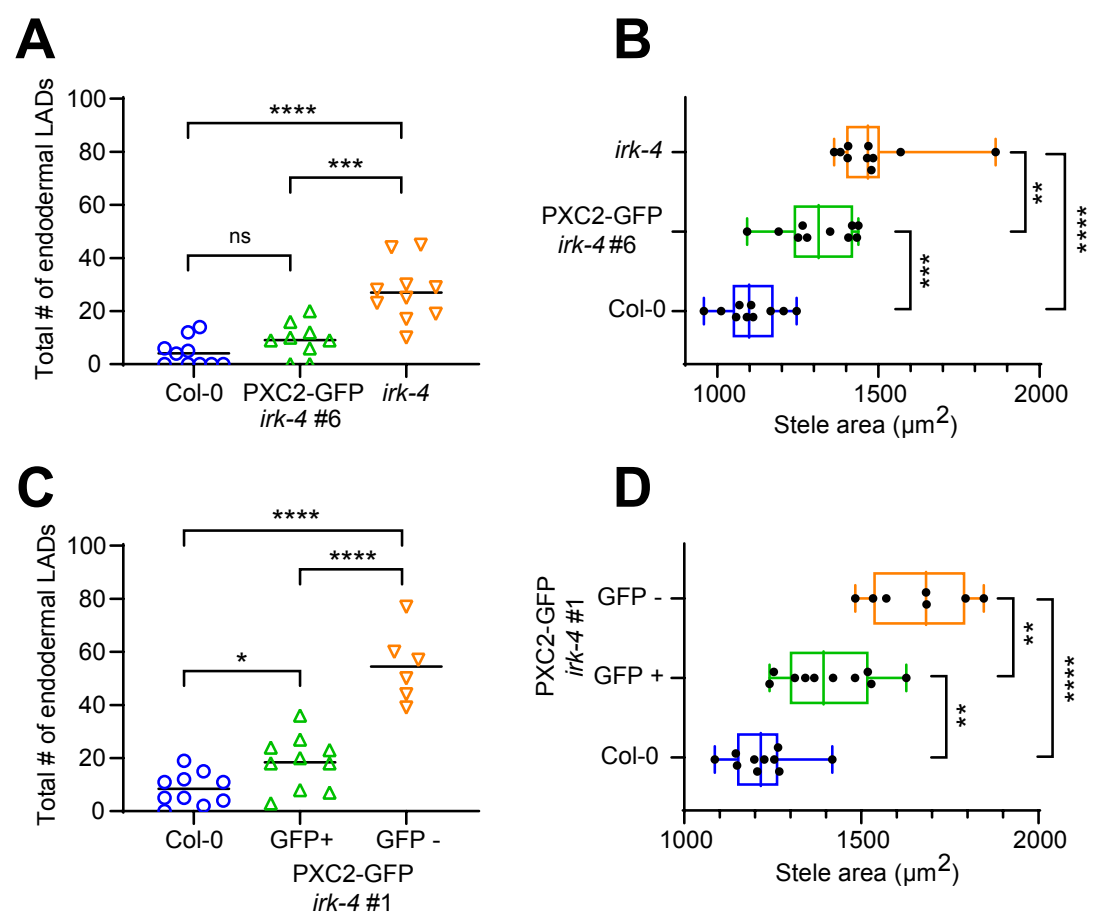

Figure 4. PXC2 partially rescues abnormal root phenotypes in irk. Graphs showing total number of LADs and stele area in (A, B) Col-0, irk-4 pSCR:PXC2:GFP, and irk-4 plants (at $4 \mathrm{dps}$, 9-10 roots per genotype) and (C, D) Col-0 and irk-4 plants segregating for $p S C R: P X C 2: G F P$ (at $6 \mathrm{dps}$, Col-0 $\mathrm{n}=10$, GFP $+n=10$, GFP-, $n=6-7$ ). All data shown are from a single, representative biological replicate of $\geq 3$. Scatter plots $(A, C)$ : Total number of LADs in individual roots indicated by colored symbols with the black bar indicating the mean for each data set. Box plots $(B, D)$ : whiskers indicate $\mathrm{min} / \mathrm{max}$ with interquartile range and mean shown in colored boxes/lines, respectively, and black dots indicate measurements for individual roots. Abbreviations: $\mathrm{ns}=$ not significant, ${ }^{*}=\mathrm{p}$ value $<0.05$, ${ }^{* *}=p$ value $<0.01,{ }^{* * *}=p$ value $<0.001,{ }^{* * *}=p$ value $<0.0001$, Student's t-test. 
Figure 5

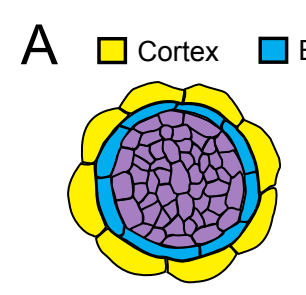

Wild type

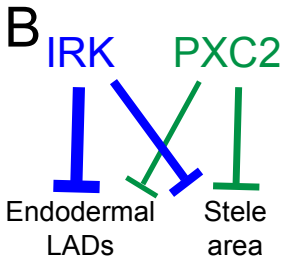

Normal stele area, 8 endodermal cells
Endodermis

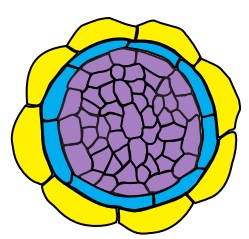

pxc2

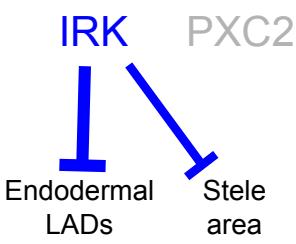

Enlarged stele area, 8 endodermal cells

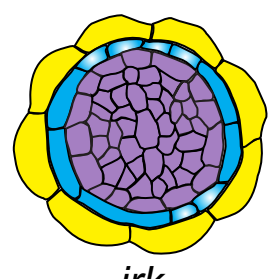

irk

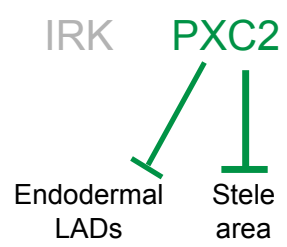

Increased stele area \& endodermal cells

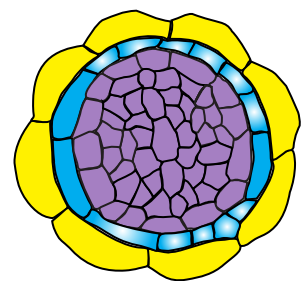

irk pxc2

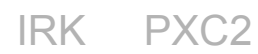

Endodermal Stele LADs area

Enhanced stele area \& endodermal cell increases

Figure 5. Summary IRK and PXC2 function to restrict stele area and endodermal LADs and phenotypic outcomes. (A) Schematic representations of transverse sections of the root showing phenotypes observed in endodermal cells and stele in Col-0, $p x c 2$, irk, and irk pxc2. Highlighted endodermal cells indicate the occurrence of LADs. (B) Diagrams illustrating the relative roles of IRK and PXC2 in each genotype with regard to restriction of stele area and endodermal LADs. Weight of the lines indicates the proposed contribution of each protein to the processes with the phenotypic outcomes listed below each. 
Figure S1
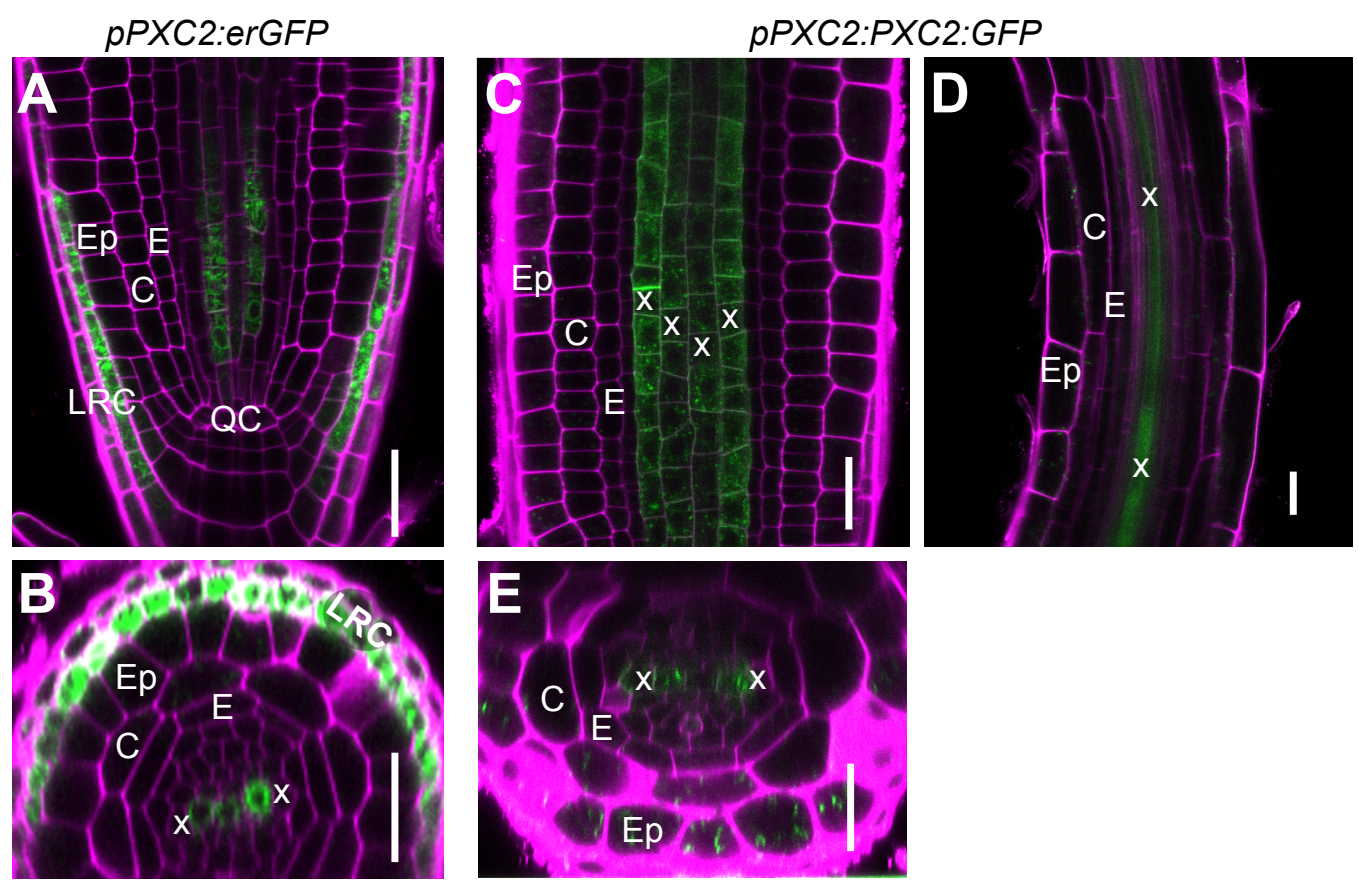

Figure S1. PXC2 promoter activity and PXC2-GFP accumulation across the root developmental zones. (A-E) Confocal micrographs of roots with the propidium iodide (PI) channel (magenta) and GFP channel (green) merged. (A) Median longitudinal and (B) transverse optical sections showing pPXC2:erGFP activity in the meristematic zone. (C, D) Median longitudinal sections showing PXC2-GFP accumulation when driven by $p P X C 2$ in xylem cell types in the $(C)$ meristematic and $(D)$ elongation zones. (E) Transverse optical section in the distal root meristem expressing $p P X C 2: P X C 2: G F P$. Scale bars: $25 \mu \mathrm{m}$. Abbreviations: (QC) quiescent center, (E) Endodermis, (C) Cortex, (Ep) Epidermis, (LRC) Lateral Root Cap, (x) xylem axis. 
Figure S2

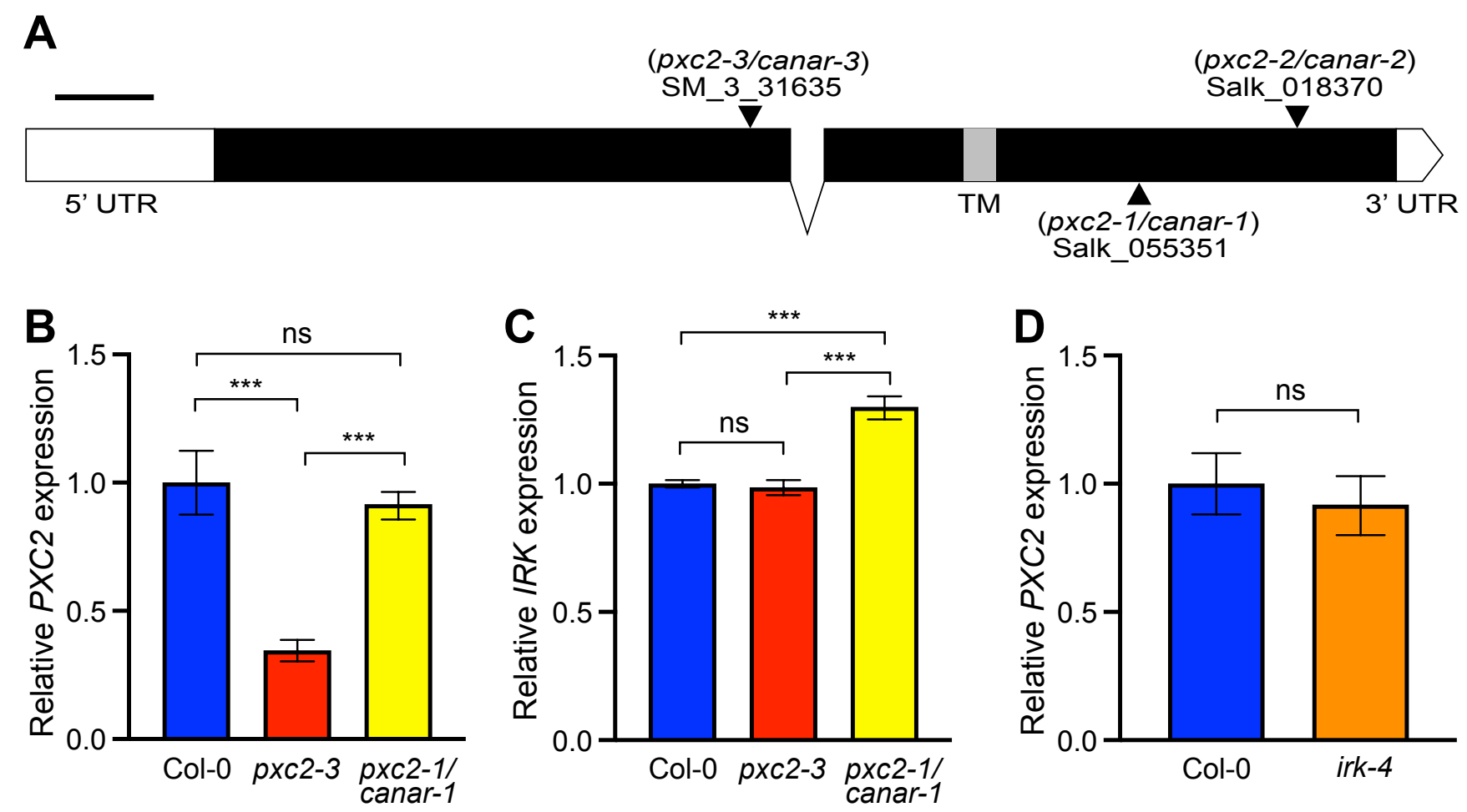

Figure S2. PXC2 gene structure and expression analyses. (A) PXC2 (At5g01890) gene structure with positions of insertional alleles indicated (triangles) and the region encoding for the transmembrane (TM) domain highlighted in gray. Scale bar: 250 base pairs. (B-D) Expression of $P X C 2$ and IRK relative to controls. (B, C) Col-0, pxc2-3, and pxc2-1/canar-1 or (D) Col-0 and irk-4 samples. Representative data shown from a single biological replicate (of three) with error bars showing standard deviation among three technical replicates. Primers for QRT-PCR on PXC2 are located on either side of the intron and are downstream of $p x c 2-3$ insertion site (Table S1). Abbreviations: ns - not significant, ${ }^{* * *}=p$-value $<0.001$, Student's t-test. 
Figure S3
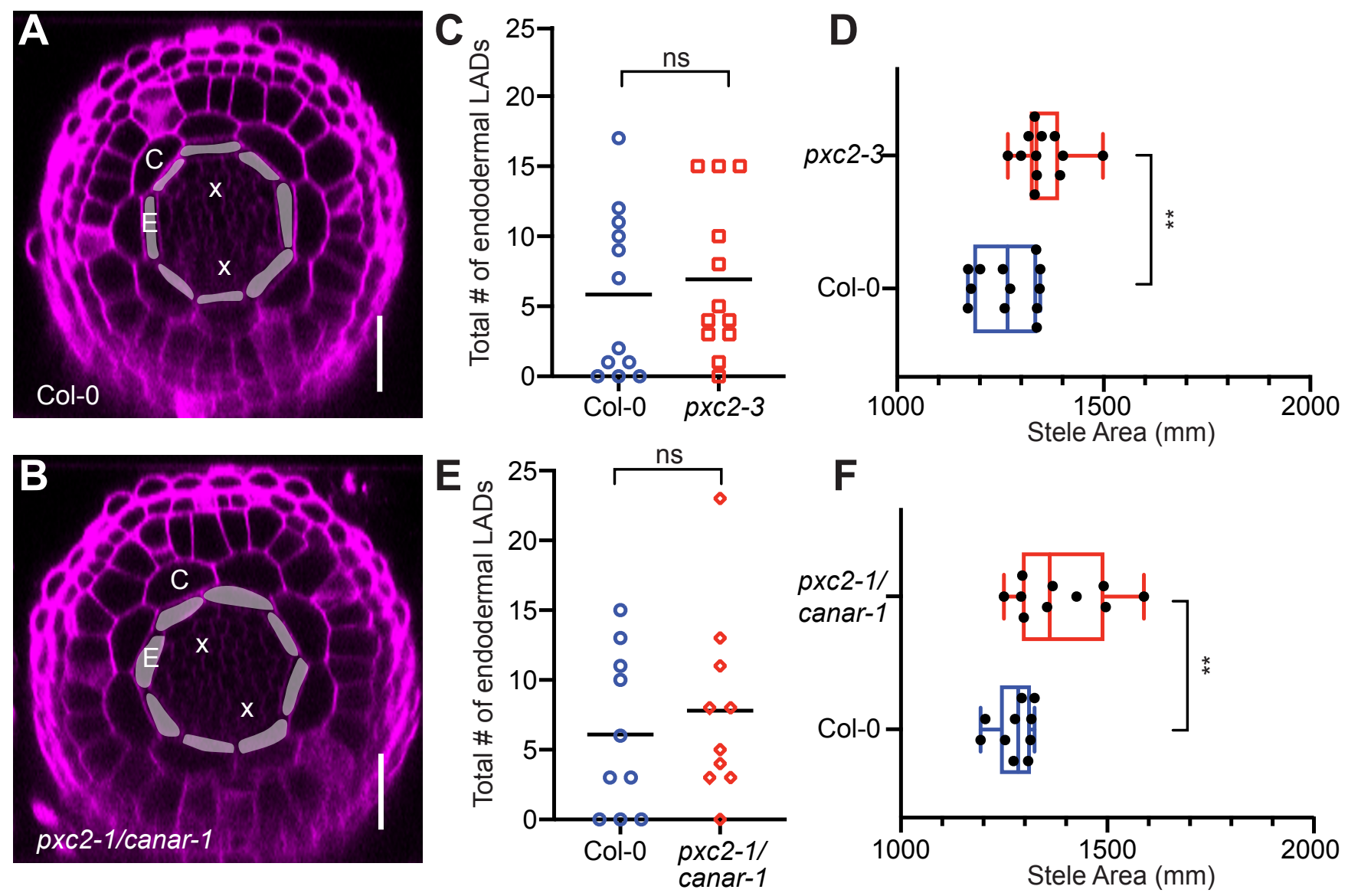

Figure S3. pxc2-3 and pxc2-1/canar-1 roots have similar abnormal root phenotypes. (A, B) Confocal micrographs of transverse sections from root meristems stained with $\mathrm{PI}(\mathrm{A})$ Col-0 and (B) pxc2-1/canar-1 at 6 dps with endodermal cells shaded. (C-F) Graphs showing total endodermal LAD and stele area in Col-0 (C, D) and pxc2-3 or (E, F) pxc2-1/canar-1 roots at $6 \mathrm{dps}$. Data shown from a single representative biological replicate of $\geq 3$ with data in $(C, D)$ shown in Figure 2 with rescaled $y$-axis in (C) for comparison with pxc2-1/canar-1 data. $(E, F) n=10$ roots per genotype. Scatter plots $(C, E)$ : Total number of LADs in individual roots represented by colored symbols with the black bar indicating the mean for each data set. Box plots (D, F): whiskers indicate $\mathrm{min} / \mathrm{max}$ with interquartile range and mean shown in colored boxes/lines, respectively, and black dots indicate measurements for individual roots. Scale bar: $25 \mu \mathrm{m}$. Abbreviations: $\mathrm{C}$ - cortex, $\mathrm{E}-$ endodermis, $\mathrm{x}-\mathrm{xylem}$ axis, $\mathrm{ns}=$ not significant, ${ }^{* *}=p$ value $<0.01$, Student's t-test. 

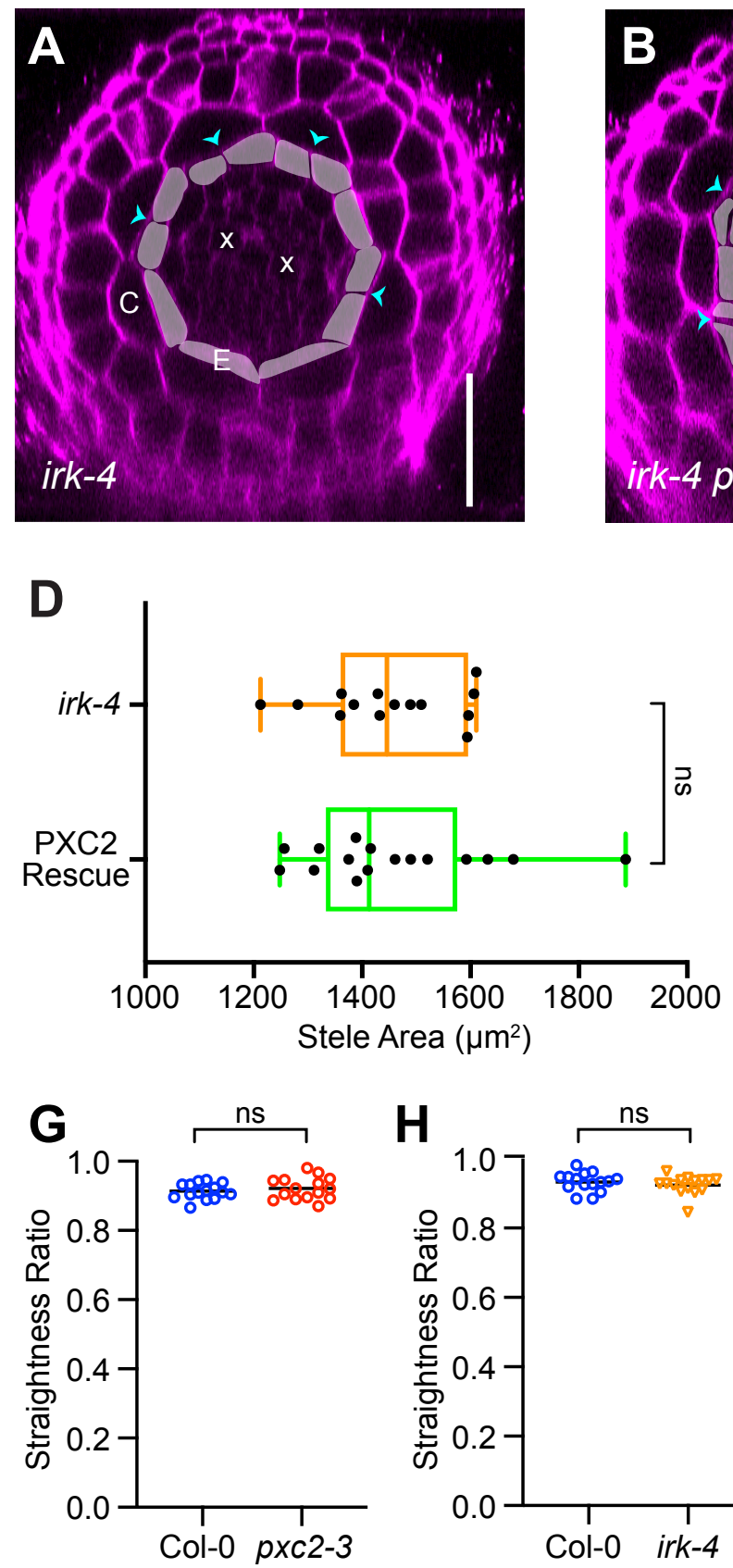
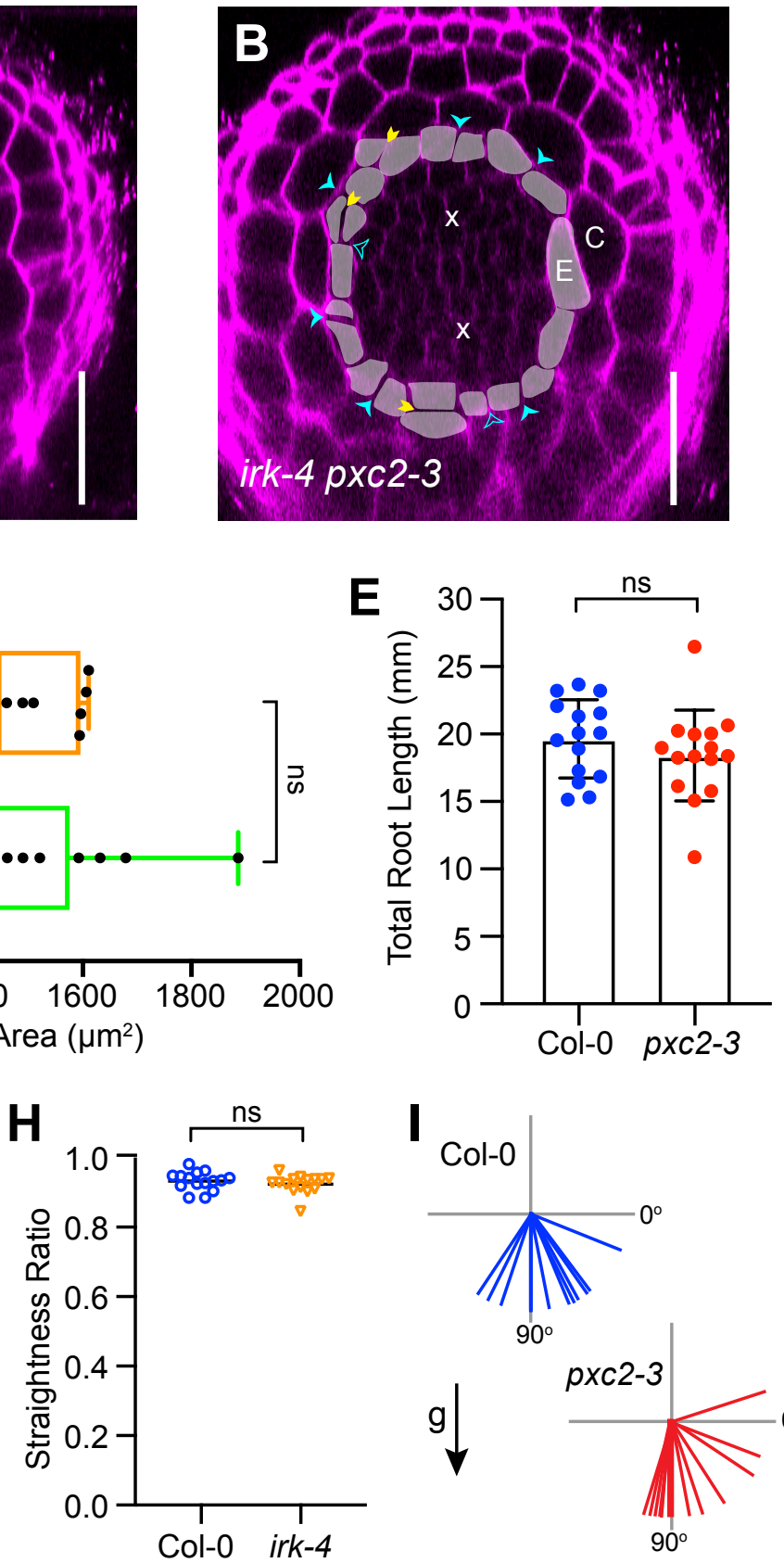
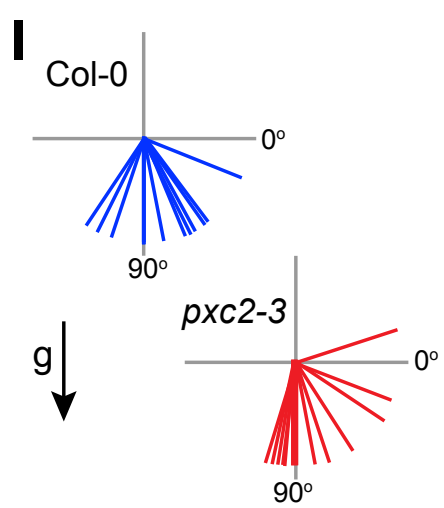
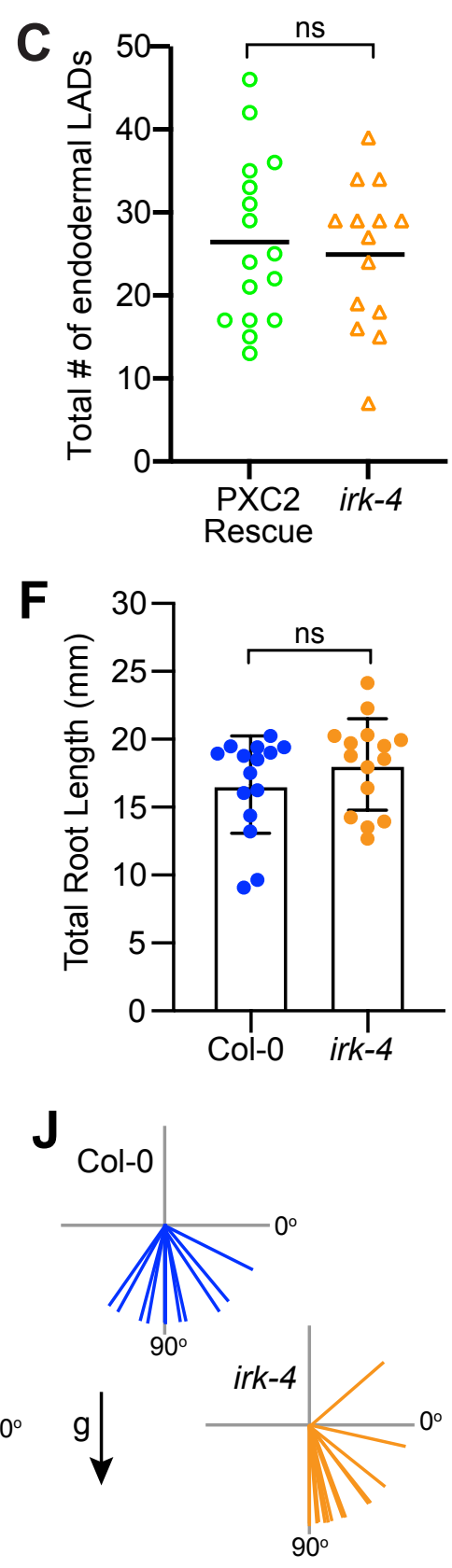

Figure S4. Disorganization of irk-4 pxc2-3 roots is rescued back to irk-4 by expression of pPXC2:PXC2:GFP and pxc2-3 and irk-4 single mutant root growth phenotypes. $(A, B)$ Confocal micrographs of transverse optical sections from root meristems stained with $\mathrm{PI}(\mathrm{A})$ irk-4 and (B) irk-4 pxc2-3 at 6 dps with endodermal cells shaded. Cyan arrowheads indicate LADs, yellow arrowheads indicate periclinal divisions, and cyan outlined arrowheads indicate possible, but uncertain, endodermal LADs based on cell position. Scale bars: $25 \mu \mathrm{m}$. Note the double mutant phenotype is more severe at 6 dps than 4 dps (compare to Figure 2F). (C, D) At 4 dps, expression of $p$ PXC2:PXC2:GFP in irk-4 pxc2-3 (PXC2 Rescue) rescues (C) endodermal LADs and (D) stele area back to the irk-4 phenotype. (PXC2 Rescue: $n=16$, irk-4 $n=14$ ). Data from a single representative biological replicate of $\geq 3$. Box plot (D): whiskers indicate $\mathrm{min} / \mathrm{max}$ with interquartile range and mean shown in colored boxes/lines, respectively, and black dots indicate measurements for individual roots. Graphs of root growth and phenotypes in Col-0 and pxc2-3 or irk-4 roots at $7 \mathrm{dps}(\mathrm{E}, \mathrm{F})$ Total root length, $(\mathrm{G}, \mathrm{H})$ straightness ratio, and (I, J) Root tip angle $8 \mathrm{~h}$ after gravistimulation by turning plates $90^{\circ}$. Colored lines $=$ angle of individual root tips. (E-J) $n=15$ per genotype. New gravity vector $\left(90^{\circ}\right)$ indicated by $g$ and black arrow, previous gravity vector aligned with $0^{\circ}$. Scatter plots $(C, G, H)$ : Data for individual roots indicated by colored symbols with the black bar indicating the mean for each data set. Error bars = standard deviation. All data shown is from a single biological replicate of $\geq 3$. Student's t-test. Abbreviations: $\mathrm{C}$ - cortex, $\mathrm{E}$ - endodermis, $\mathrm{x}$ - xylem axis, ns = not significant. 


\section{Table S1}

\section{Primer Purpose}

Gene/Region of interest (AGI/name)
Primer sequence (5'-3')

\section{Notes}

TCAAGCAATCAACTCTCTGGG

pxc2-3

SM_3_31635 RP

TGGTGTTGAAGAAACCTCCAG

pxc2-3

Spm32

TACGAATAAGAGCGTCCATTTTAGAGTGA

CAATCTCTCGGGAAGTCTTCC

ССТTСТСТСАССGTCTCACAC

ATTTTGCCGATTTCGGAAC

LBb1.3

\section{Cloning $\quad$ PXC2, At5g01890}

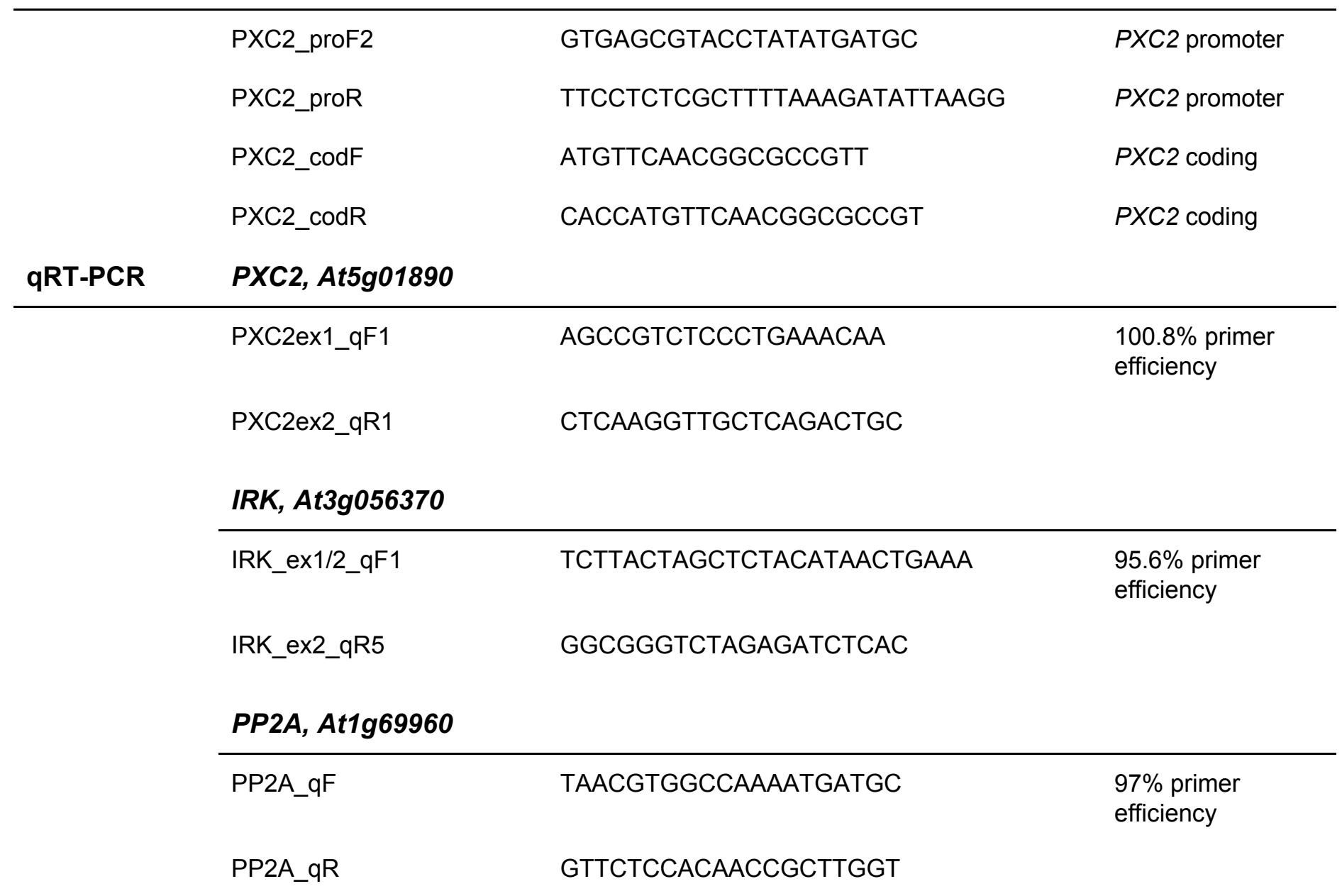

